\title{
Aprendiendo a través de Tareas de Evaluación Auténticas: Percepción de Estudiantes de Grado en Educación Infantil
}

\section{Learning through Authentic Assessment Tasks: Perception of Early Childhood Education Undergraduates}

\author{
Jaione Cubero-Ibáñez * \\ Nicolás Ponce-González \\ Universidad de Cádiz, España
}

\begin{abstract}
La evaluación auténtica ofrece a los estudiantes oportunidades para aprender a través del propio proceso de evaluación (Brown, 2015). Uno de los principios fundamentales para lograrlo es desarrollar tareas de evaluación auténticas que representen tanto el contenido como las habilidades centrales (Vallejo y Molina, 2014). El estudio que se presenta sigue un diseño de encuesta basado en cuestionario con el objetivo de conocer la percepción de los estudiantes universitarios acerca del proceso de evaluación llevado a cabo en la asignatura Observación Sistemática y Análisis de Contextos, diseñado a partir de las propuestas de Boud (2010). La muestra se compone de 131 estudiantes, pertenecientes a los tres grupos de primer curso del Grado en Educación Infantil (Universidad de Cádiz). Los resultados muestran un alto grado de satisfacción por parte de los estudiantes con la evaluación llevada a cabo. Perciben que la tarea de evaluación les ha permitido aumentar la motivación y comprender la observación sistemática como una técnica necesaria para la reflexión sobre la práctica educativa, además de ser realista y retadora lo cual ha facilitado un aprendizaje más útil y significativo. Aprender a través de una experiencia real se convierte en el primordial aspecto positivo, destacando la falta de tiempo como principal inconveniente. El diseño y puesta en práctica de este tipo de evaluaciones rompen con el sistema de evaluación predominante en las universidades, más dirigida a la comprobación y valoración de los aprendizajes que a la mejora de estos (Lukas, Santiago, Lizasoain y Etxeberria, 2017).
\end{abstract}

Palabras clave: Evaluación; Aprendizaje; Evaluación formativa; Retroalimentación; Educación superior.

\begin{abstract}
Authentic assessment offers students opportunities to learn through the process of assessment itself (Brown, 2015). One of the key principles for the design of this type of assessment is developing authentic assessment tasks that represent both content and the core skills (Vallejo y Molina, 2014). This study follows a questionnaire-based survey design with the goal of finding out university students' perception of the assessment process conducted in the subject Systematic Observation and Context Analysis, based on the proposals of Boud (2010). The sample consists of 131 students, belonging to the three first-year groups on the Degree in Early Childhood Education (University of Cádiz). The results show a high level of satisfaction among the students with the assessment conducted. They perceive that the assessment task allowed them to increase motivation and understand systematic observation as a necessary technique for reflection on educational practice, as well as being realistic and challenging, which has facilitated more useful and meaningful learning. Learning through a real experience is the main positive aspect, with the lack of time highlighted as the main drawback. The design and implementation of these types of assessment break away from the predominant assessment system at universities, more aimed at the verification and evaluation of learning than at its improvement (Lukas, Santiago, Lizasoain y Etxeberria, 2017).
\end{abstract}

Keywords: Assessment; Learning; Formative assessment; Feedback; Higher education.

*Contacto: jaione.cubero@uca.es

issn: 1989-0397

www.rinace.net/riee/

https://revistas.uam.es/riee
Recibido: 2 de enero de 2020

$1{ }^{\text {a }}$ Evaluación: 2 de marzo de 2020

$2^{\text {a }}$ Evaluación: 16 de marzo de 2020

Aceptado: 23 de marzo de 2020 


\section{Introducción}

Hace ya diez años, Boud (2010) redactaba el documento "Assessment 2020" donde se recogen un conjunto de propuestas para la reforma de la evaluación en Educación Superior con la intención de afrontar cambios sustanciales en un contexto de evolución global. Sin embargo, estudios recientes demuestran que las prácticas evaluativas en las universidades españolas se basan en enfoques tradicionales (Panadero, Fraile, Fernández, CastillaEstévez y Ruiz, 2018), poniendo el énfasis en la comprobación y valoración de los aprendizajes frente a la mejora de estos (Lukas, Santiago, Lizasoain y Etxeberria, 2017). A pesar de advertirse algunos cambios en la forma de evaluación, el fondo sigue representando una herramienta principalmente acreditativa, presentándose como un elemento prioritariamente final y asociado usualmente a exámenes y sensaciones negativas (Bilbao y Villa, 2019). Rodríguez-Gómez, Ibarra-Sáiz y Cubero-Ibáñez (2018) constatan una baja participación de los estudiantes en los procesos de evaluación y destacan la falta de tareas de evaluación que permita a los estudiantes responder de forma creativa "impidiéndoles gestionar y ejecutar estrategias educativas para la formación autónoma” (p. 198).

Este panorama demuestra la discordancia existente entre las principales tendencias y enfoques de evaluación alternativa que abogan por un aprendizaje estratégico y a lo largo de la vida (Boud, 2000; Carless, Joughin y Mok, 2006; Rodríguez-Gómez e Ibarra-Sáiz, 2011, 2015), con las prácticas evaluativas imperantes. Por ello, es importante analizar y dar a conocer aquellas experiencias, al parecer escasas, desarrolladas bajo enfoques alternativos de evaluación en el ámbito universitario, para que los profesionales aprendan de otras prácticas y puedan poner en marcha las innovaciones que se hayan demostrado que funcionan bien (Brown, 2015).

Este trabajo parte de la importancia del diseño de una evaluación auténtica para ofrecer a los estudiantes oportunidades para aprender a través del propio proceso de evaluación (Brown, 2015), teniendo en cuenta que uno de los principios fundamentales para lograrlo es el diseño y desarrollo tareas de evaluación auténticas que representen tanto el contenido como las habilidades centrales de los estudiantes (Vallejo y Molina, 2014).

La evaluación auténtica no es un concepto nuevo, pues ya tuvo gran discusión y consideración hace algunas décadas (Herrington y Herrington, 2006), pero, a pesar de mantener una base teórica importante y un discurso sólido y creciente, su práctica ha sido débil e incipiente (Ahumada, 2005). Siguiendo a Vallejo y Molina (2014), este enfoque de evaluación establece un vínculo entre lo conceptual y procedimental y tiene como base la coherencia entre los objetivos de aprendizaje y de evaluación, utilizando una retroalimentación constructiva para poder informar y crear conciencia en el alumnado sobre el modo en que progresan. En este sentido, la evaluación auténtica alienta a los estudiantes a enfrentarse a prácticas desafiantes, proporcionando oportunidades, a través de las formas en que estos participan en los procesos de evaluación, para que aprendan y reflexionen sobre su aprendizaje favoreciendo así su desarrollo personal y profesional (Vu y Dall, 2014).

Para poder llevar a cabo este tipo de prácticas en las aulas universitarias, Ibarra-Sáiz y Rodríguez-Gómez (2014), proponen diez principios básicos que pueden guiar una evaluación auténtica en la Educación Superior. Entre ellos se destacan aspectos relevantes como credibilidad, diálogo, mejora, participación, reflexión, regulación, reto, 
sostenibilidad, transparencia y transversalidad. Brown (2015) en su manifiesto sobre cómo debe ser una evaluación auténtica, coincide con algunos de los principios mencionados anteriormente, poniendo el énfasis en que esta debe:

- Estar orientada a la acción, con estudiantes que aprenden a través de la acción en un rango de contextos relevantes

- Ser representativa del esfuerzo y del rendimiento del estudiante, con criterios ponderados en función de su importancia en términos de los resultados del aprendizaje

- Garantizar una retroalimentación puntual en su ejecución (para que los estudiantes tengan la oportunidad de aprender de la retroalimentación de una tarea antes de entregar otra)

- Habilitar un diálogo productivo entre profesores universitarios y estudiantes

- Ser verdaderamente útil para el progreso de los estudiantes, consistente en su asesoramiento y de gran apoyo, sin dejar de ayudar a los estudiantes a entender lo que tienen que hacer para progresar.

Otros nuevos enfoques han venido a complementar la esencia del término evaluación auténtica, como son la evaluación orientada al aprendizaje (Carless, Joughin y Mok, 2006) y la evaluación como aprendizaje y empoderamiento (Rodríguez-Gómez e Ibarra-Sáiz, 2015). Desde nuestro punto de vista todos ellos son enfoques de evaluación alternativos y auténticos, con diferentes focos de atención, considerando el enfoque de evaluación como aprendizaje y empoderamiento el más completo, entendiéndose la evaluación como una oportunidad de aprendizaje que por sí misma permite el empleo de estrategias y desarrollo de competencias transferibles que fomenten la autoconciencia de las necesidades formativas, la autorregulación del proceso de aprendizaje, el aprendizaje autónomo a lo largo de la vida y la autodeterminación en la toma de decisiones (Rodríguez-Gómez e Ibarra-Sáiz, 2015).

Como se puede apreciar en la figura 1, las tareas auténticas deben reunir un conjunto de características para ser reconocidas como tal.

Siguiendo el enfoque de la evaluación como aprendizaje y empoderamiento, las tareas de evaluación de los estudiantes deben ser tareas de "calidad" entendiéndose por aquellas "actividades diseñadas con el fin de valorar la capacidad de los estudiantes para aplicar y utilizar sus competencias, conocimientos y destrezas en desafíos del mundo real" (Rodríguez-Gómez e Ibarra-Sáiz, 2015, p. 21).

Estas son caracterizadas por Ibarra-Sáiz, Rodríguez-Gómez y García-Jiménez (2016) como aquellas que:

- Presentan una naturaleza significativa: realistas, auténticas en el ámbito profesional, retadoras, funcionales y útiles, que desarrollen mediante su ejecución un conjunto de competencias transversales y sofisticadas relevantes para el futuro.

- Exigen profundidad: conocimiento consistente de contenidos de la disciplina y la puesta en práctica de un pensamiento analítico y reflexivo de alto de nivel. 
- Y requieren demostraciones: evidencias tangibles y directas (productos y actuaciones), cuyo valor está no solo en el producto, sino en el proceso de ejecución en sí mismo.

- Centrarse en los procesos (no sólo productos).

- Ser realistas, retadoras, diversas, globales.

- Ser relevantes, útiles, significativas, complejas.

- Integrar diferentes competencias, destrezas, habilidades y conocimientos.

- Implicar diferentes tipos de aprendizaje y actuaciones de forma que faciliten un aprendizaje útil, significativo y estratégico.

- Facilitar el empoderamiento del estudiante sobre su propio aprendizaje y evaluación.

- Permitir la construcción del conocimiento y su demostración en contextos reales.

- Evaluar al estudiante en los contextos profesionales reales.

- Posibilitar la participación en el proceso de evaluación.

- Facilitar la proalimentación.

- Utilizar múltiples medios e instrumentos de evaluación.

- Proximidad ecológica.

Figura 1. Características de las tareas auténticas Fuente: Rodríguez-Gómez e Ibarra-Sáiz (2011, p. 85).

Por lo tanto, la puesta en práctica de una evaluación como aprendizaje exige enfrentar a los estudiantes a tareas complejas, auténticas, de calidad y fomentar la participación de los mismos en los procesos de evaluación, utilizando la retroalimentación y proalimentación como estrategias para proporcionar oportunidades para aprender a mejorar sus tareas (ver figura 2), con la finalidad de que éstos sean capaces de autorregular su aprendizaje y puedan transferir las habilidades desarrolladas a otros contextos extra académicos (Cubero-Ibáñez, 2017).

Retroalimentación
- El profesor, como un
miembro más de la
comunidad de práctica de
un estudiante, ofrece
comentarios u
orientaciones a un
estudiante, a un grupo o
al conjunto de la clase,
para indicar cómo deben
reducir la distancia que
separa la calidad de sus
actuaciones o productos
de lo que el profesor
considera una actuación
o producto de calidad.
- Liderado por el profesor

Proalimentación
- El profesor, como un
miembro más de la
comunidad de práctica
del estudiante, facilita el
diálogo con el estudiante
para que éste utilice toda
la información y decida
cómo regular su
aprendizaje hasta
alcanzar el nivel de
referencia fijado para una
actuación o producto
presente o futuro.
- Compartido entre
profesor y estudiante

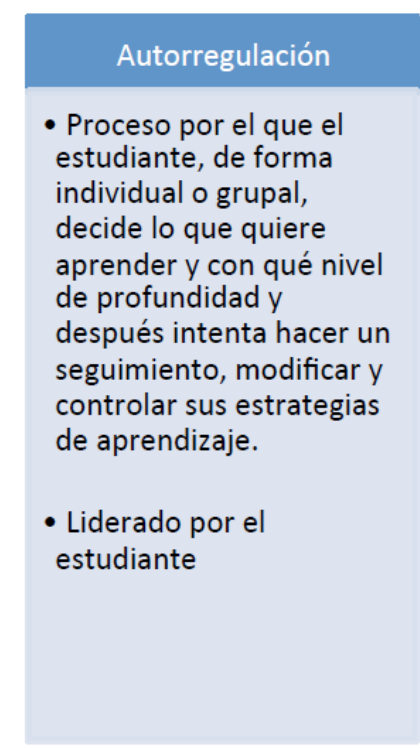

Figura 2. Definición de retroalimentación, proalimentación y autorregulación Fuente: García-Jiménez, Ibarra-Sáiz y Rodríguez-Gómez (2013, p. 9). 
El objetivo general de este estudio se centra en conocer la percepción de los estudiantes universitarios acerca del proceso de evaluación llevado a cabo en la segunda parte de la asignatura Observación Sistemática y Análisis de Contextos (OSA). Como objetivos específicos, se persigue:

- Conocer la percepción de los estudiantes acerca de la tarea de evaluación "Elaboración grupal de un informe de observación sistemática aplicada en un centro/contexto de Educación Infantil”

- Comprobar el grado de satisfacción de los estudiantes con la evaluación de la segunda parte de la asignatura

- Identificar aspectos positivos, negativos y propuestas de mejora, por parte de los estudiantes, acerca de la experiencia realizada

\section{Método}

\subsection{Contexto y procedimiento}

La investigación se contextualiza en la asignatura "Observación Sistemática y Análisis de Contextos", impartida en el primer curso del Grado en Educación Infantil de la Universidad de Cádiz. Esta asignatura es compartida por dos áreas de conocimiento, llevándose a cabo esta experiencia en la segunda parte perteneciente al área de Métodos de Investigación y Diagnóstico en Educación. De los dos docentes del área implicados en la asignatura, el docente 1, coordinador de la asignatura y con experiencia y formación en evaluación como aprendizaje en Educación Superior, impartió a los grupos A y C y el docente 2 , con algo menos de experiencia y formación, al grupo B.

Desde los inicios de la asignatura, con la introducción del plan Bolonia, se ha ido modificando el diseño de la misma, adaptándola a las necesidades detectadas a través de la experiencia docente, así como mediante la realización de diferentes estudios relacionados con la propia práctica educativa (Gómez y Quesada-Serra, 2017; Quesada-Serra, Gómez, Gallego y Cubero-Ibáñez, 2019), siempre con la finalidad de favorecer el aprendizaje de los estudiantes a través de la evaluación.

El diseño de la evaluación de esta segunda parte de la asignatura, tomó como punto de partida las propuestas para la reforma de la evaluación en Educación Superior "Asessessment 2020" de Boud (2010), resumidas en la figura 3, y los principios de la evaluación en la Educación Superior recogidos por Ibarra-Sáiz y Rodríguez-Gómez (2014), nombrados previamente.

El proceso de evaluación llevado a cabo (ver figura 4), enmarcado en el enfoque de la evaluación como aprendizaje y empoderamiento, pone el énfasis en dos tareas de evaluación, las cuales fueron diseñadas teniendo en cuenta las características de las tareas de evaluación auténticas (Rodríguez-Gómez e Ibarra-Sáiz, 2011), y de calidad (Ibarra-Sáiz y Rodríguez-Gómez, 2015), citadas en el anterior apartado. Ambas tareas mantienen una estrecha relación entre sí, ya que las dos están enfocadas al diseño de una observación sistemática en un contexto educativo, pero presentan características diferenciadas. 


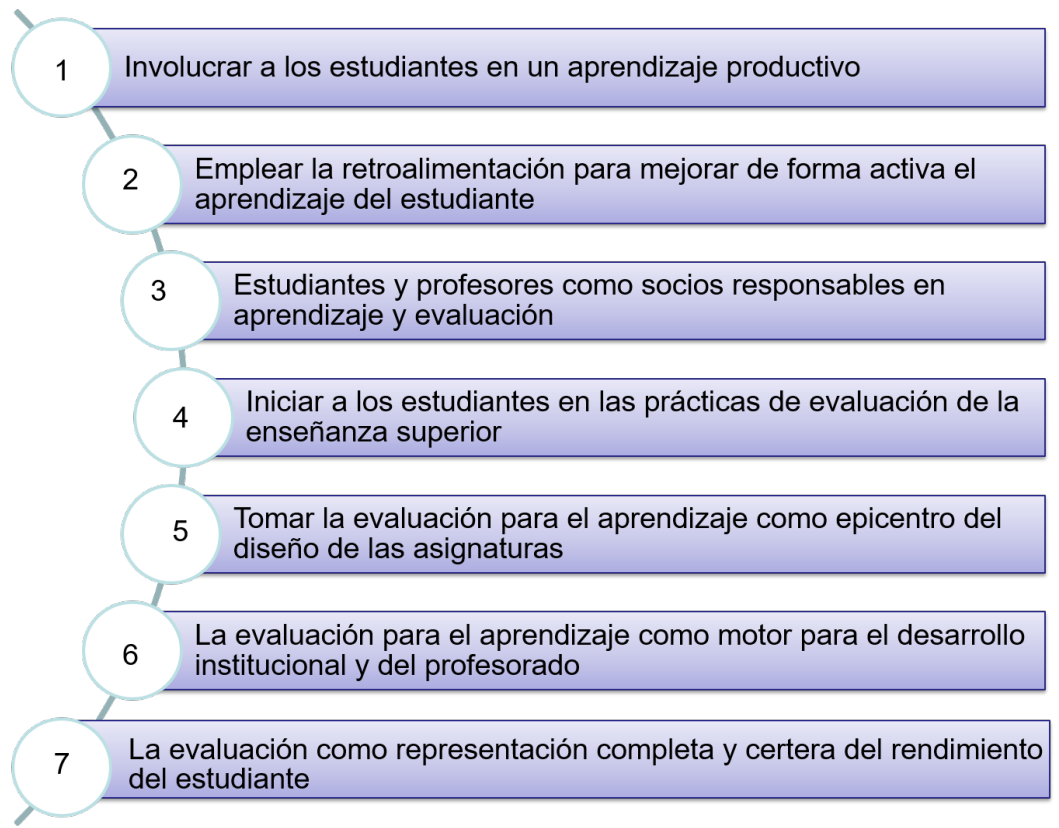

Figura 3. Propuestas para la reforma de la evaluación en Educación Superior Fuente: Elaboración propia a partir de Boud (2010).

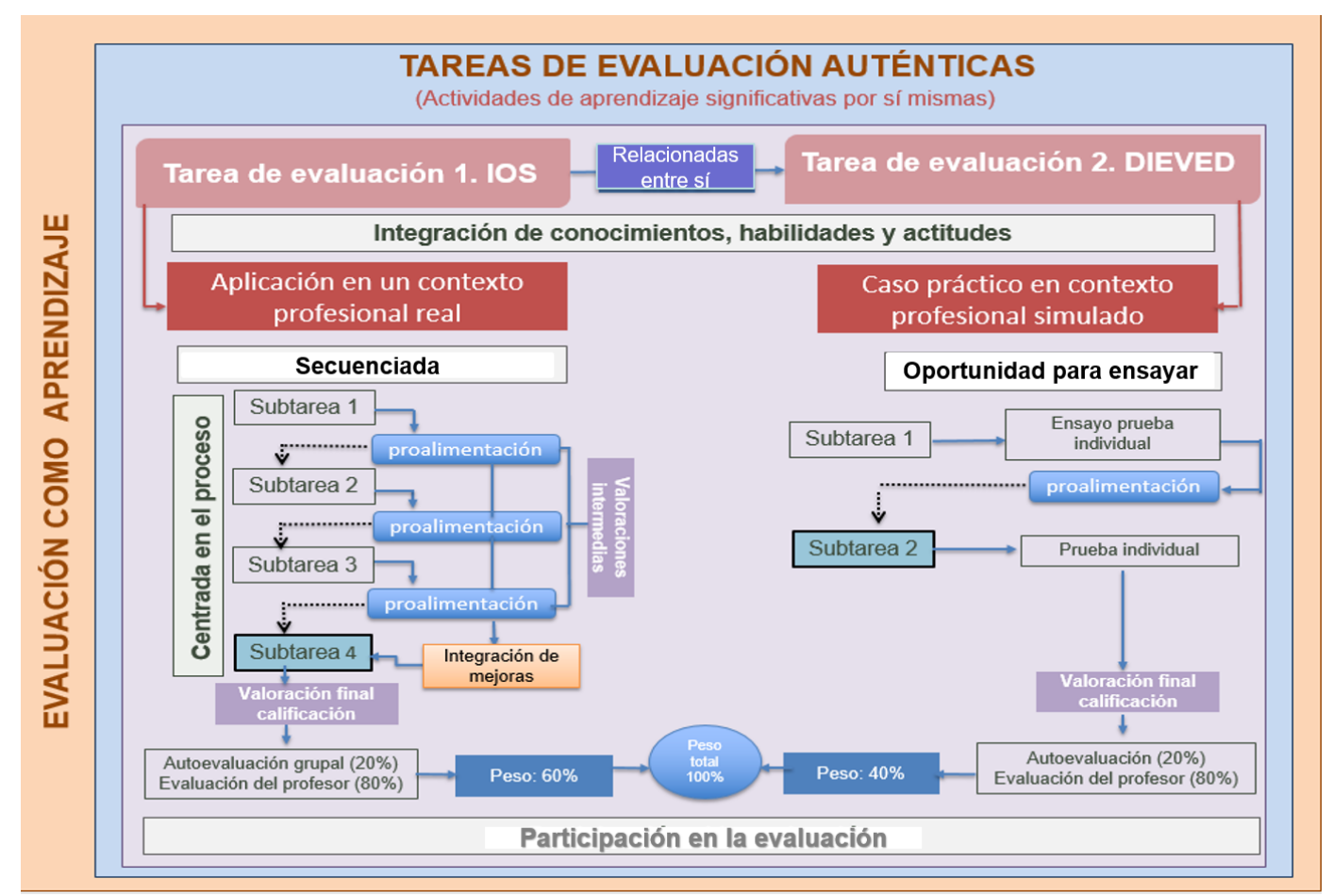

Figura 4. Proceso de evaluación de la segunda parte de la asignatura OSA, curso 20182019

Fuente: Elaboración propia.

La primera tarea de evaluación, elaboración de un Informe de observación sistemática aplicada en un centro/contexto de Educación Infantil "IOS”, de carácter grupal, se aplica en un contexto educativo real y se realiza progresivamente en el tiempo, de forma secuenciada, a través de diferentes subtareas. Se ha puesto el énfasis en la proalimentación 
para el aprendizaje a lo largo de todo el proceso, entendiendo este concepto como una estrategia dialógica que aporta información, útil, relevante y prospectiva, que pueda ser utilizada por el estudiante tanto de forma inmediata como a medio y largo plazo, favoreciendo así el aprendizaje a lo largo de vida (García-Jiménez et al., 2013). En este caso, al ser una asignatura de primer año, la información es proporcionada entre el docente y el grupo de estudiantes. Los resultados intermedios de la evaluación, no influyen en calificaciones finales de los estudiantes, sino que son utilizados para mejorar el aprendizaje, creando un diálogo entre docentes y discentes acerca de cómo mejorar la calidad de su trabajo, posibilitando la integración de las mejoras en la última subtarea, objeto de calificación.

La segunda tarea de evaluación, diseño de una evaluación educativa contextualizada en Educación Infantil “DIEVED”, de carácter individual, parte de un caso práctico (situación problema), en un contexto profesional simulado y se realiza en el aula a modo de prueba individual el día de la convocatoria oficial de examen, teniendo la oportunidad de realizar previamente una prueba similar, a modo de ensayo, que se resuelve en el aula de forma grupal. Cabe destacar que esta es una prueba de aplicación práctica, no memorística, donde los estudiantes pueden tener acceso a los materiales y contenidos trabajados a lo largo de la asignatura, así como conexión a internet.

En cuanto al peso que tienen cada una de las tareas de evaluación en la calificación de la segunda parte de la asignatura, se le atribuye mayor porcentaje a la primera de ellas por ser un proceso más complejo y extenso que requiere mayor tiempo e implicación, y en consecuencia, mayor aprendizaje.

A continuación, se describen los elementos de la tarea de evaluación "IOS" ver figura 5, en la cual ponemos el foco de atención en nuestra investigación.

Como se puede ver en la figura 5, esta tarea de evaluación se ha dividido en diferentes subtareas conectadas entre sí, las cuáles han sido cuidadosamente estructuradas, teniendo en cuenta que se trata de una asignatura de primer curso. Todas las subtareas, realizadas en equipo durante las sesiones de pequeño grupo, han seguido el mismo proceso a la hora de su elaboración: en primer lugar, los estudiantes debían leer la guía de cada subtarea antes de asistir a la sesión práctica. Posteriormente en el aula, el profesorado realizaba la presentación de la subtarea y los criterios de evaluación. Tras el trabajo grupal en el aula, la coordinación del equipo entregaba la tarea a través del campus virtual de la asignatura (al menos 24 horas antes de la siguiente sesión práctica). Los docentes revisaban las subtareas de cada grupo. Una vez en el aula el docente mantenía una reunión con cada equipo realizando comentarios y explicaciones acerca de la tarea y manteniendo un diálogo con el alumnado acerca de las percepciones sobre la misma.

Como se ha venido mencionando, el carácter de la tarea era grupal y presencial. La duración de esta segunda parte de la asignatura es de 7 semanas, a través de las cuáles se distribuyen las subtareas de la tarea IOS (ver figura 6). 


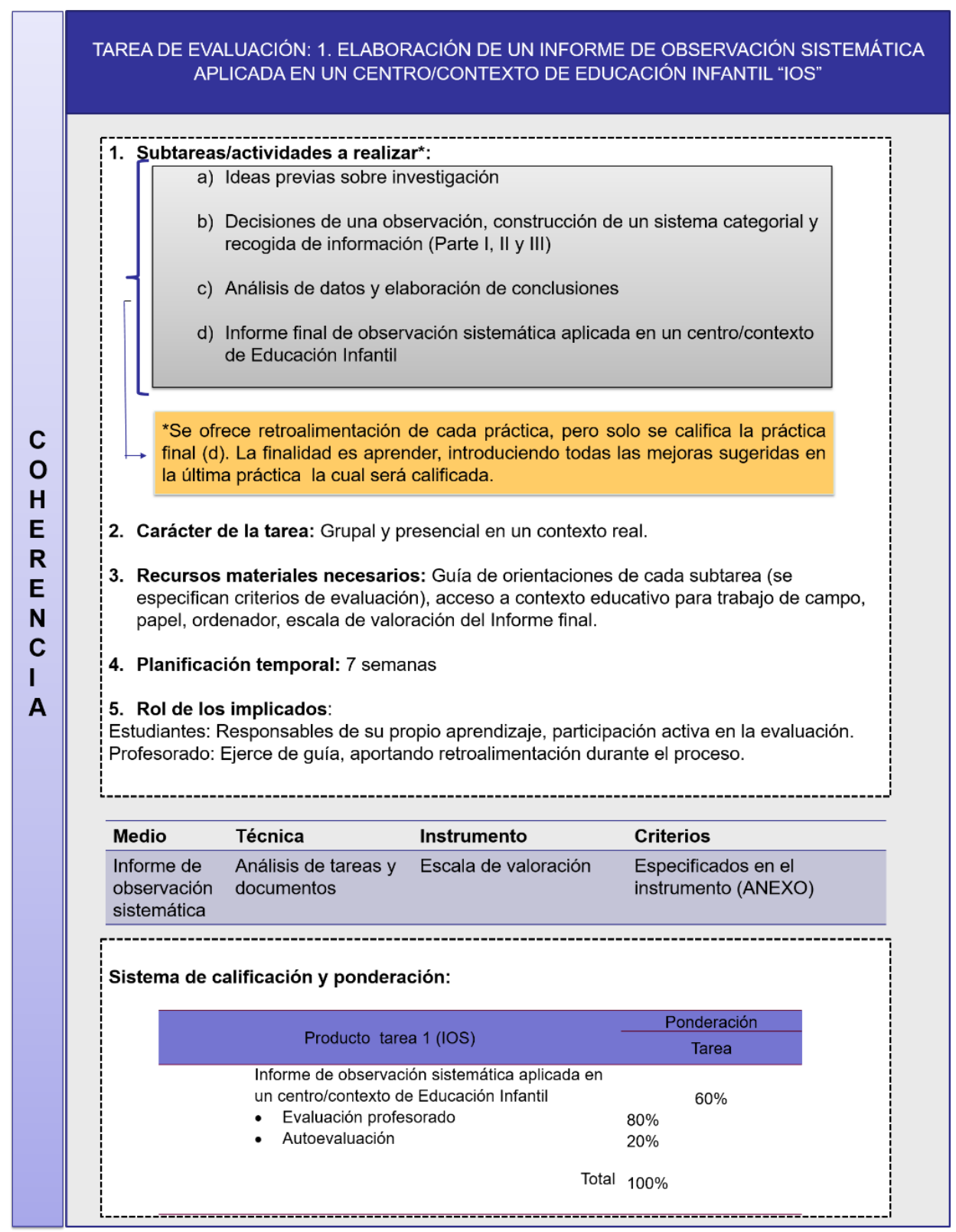

Figura 5. Descripción de los elementos de la tarea de evaluación "IOS"

Fuente: Elaboración propia a partir de Rodríguez-Gómez e Ibarra-Sáiz (2011).

En cuanto al rol de los implicados, los estudiantes jugaron un papel activo participando en la revisión y evaluación argumentada de cada una de las subtareas y en la autoevaluación grupal (calificación) de la última subtarea. A su vez, el profesorado valoró cada una de las subtareas entregadas por los estudiantes y ofreció proalimentación sobre las mismas, además de calificar la última subtarea, entendida como la suma de todas las anteriores.

Es importante destacar la importancia de la coherencia entre los diferentes elementos de la tarea de evaluación, aspecto primordial en su diseño, así como el medio, la técnica, el instrumento, y los criterios de evaluación, especificados en el Anexo I. 


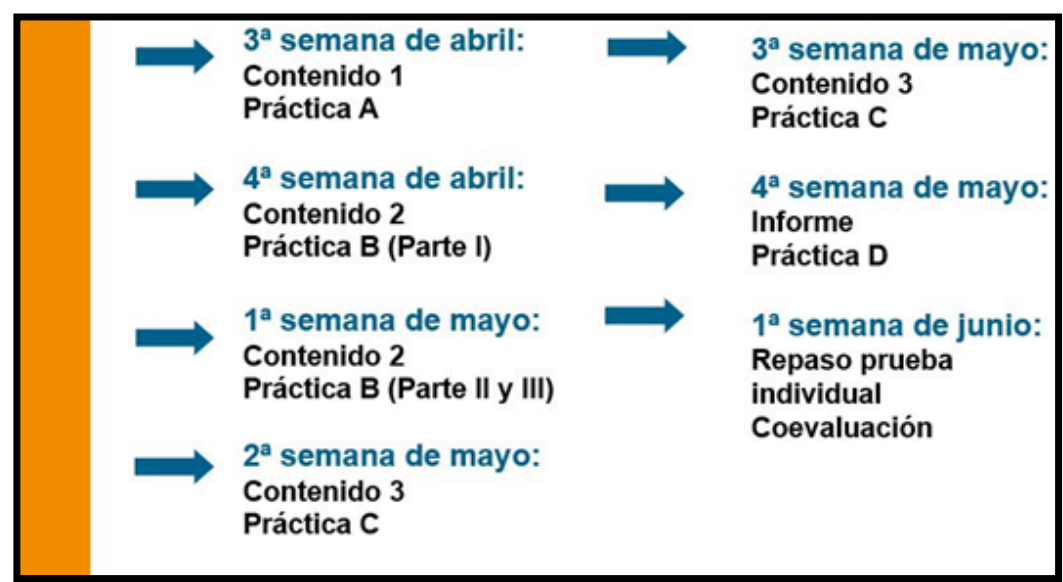

Figura 6. Planificación temporal de las subtareas de la Tarea IOS. Captura de pantalla de la presentación de la asignatura

Fuente: Elaboración propia.

\subsection{Participantes}

El estudio que se presenta sigue un diseño de encuesta basado en cuestionario. La muestra recogida durante el curso académico 2018/2019, se compone de 131 estudiantes, pertenecientes a los tres grupos de primer curso del Grado en Educación Infantil pertenecientes a la Universidad de Cádiz (A, 50 participantes [38,2\%], B 50 participantes [38,2\%] y C 31 participantes [27,3\%]), de los cuales 124 (94,7\%) son mujeres y 7 (5,3\%) son hombres. Entre los participantes, 59 (45\%) tienen menos de 21 años, 59 (45\%) tienen edades comprendidas entre 21 y 25 años, 10 (7,6\%) tienen entre 26 y 30 años y tres $(2,3 \%)$ tienen más de 30 años. Los estudiantes han accedido al grado a través de diferentes estudios de acceso a la universidad, $61(46,6 \%)$ con bachillerato, $65(49,6 \%)$ con grado superior, tres $(2,3 \%)$ con la prueba de acceso a mayores de 25 años y dos $(1,5 \%)$ con otro título universitario.

\subsection{Instrumento}

El instrumento empleado en este estudio, Cuestionario "Experiencia de Evaluación como Aprendizaje: percepción de los estudiantes EEA_(OSA)", fue diseñado específicamente para esta experiencia, tomando a Boud (2010) y Rodríguez-Gómez e Ibarra-Sáiz (2011, 2015) como principales fuentes teóricas para la elaboración del mismo. El cuestionario, construido online a través de la herramienta SurveyMonkey, se compone de 14 preguntas organizadas en 3 categorías: 1) Información general. Grupo, sexo, edad y estudios de acceso. 2) Tarea de evaluación "IOS". Valoración del nivel de acuerdo de los estudiantes con las 23 declaraciones presentadas referentes a diferentes aspectos sobre la tarea, en una escala del 1 al 10. 3) Satisfacción y mejora. Valoración de forma general del grado de satisfacción con el proceso de evaluación de la asignatura en una escala de 1 a 10 y tres preguntas abiertas sobre aspectos positivos, negativos y de mejora sobre la experiencia. Cabe señalar que el cuestionario permite ser completado sin necesidad de responder a una o varias preguntas pertenecientes a la tercera categoría. Para la respuesta del cuestionario se facilitó a los estudiantes el enlace electrónico del mismo a través de la plataforma de la asignatura una vez esta hubo finalizado, siendo contestado de forma voluntaria y anónima. La fiabilidad del cuestionario fue calculada mediante la prueba Alfa de Cronbach sobre la base del análisis de las respuestas de los 131 encuestados, destacando un elevado índice de 
consistencia interna $(0,96)$ en el promedio de las correlaciones entre los 23 ítems del instrumento.

\subsection{Análisis de datos}

El análisis se ha realizado utilizando diferentes procedimientos teniendo en cuenta la naturaleza cuantitativa o cualitativa de los datos recolectados.

Los datos cuantitativos, provenientes de las preguntas cerradas del cuestionario, se han analizado por medio del cálculo de estadísticos descriptivos como media, desviación típica y porcentaje de frecuencias de las respuestas, clasificadas en intervalos en función del nivel de acuerdo del estudiante con cada una de las declaraciones (1-4 bajo, 5-7 medio y 8-10 alto). Para la realización de dichos análisis se ha utilizado el paquete estadístico SPSS2O.

Por otro lado, se ha realizado un trabajo de carácter deductivo, por el que se ha recurrido al análisis del contenido de los datos cualitativos, procedentes de las preguntas abiertas del cuestionario, siguiendo las orientaciones procedimentales de Buendía, Colás y Hernández (1997). Los datos se han analizado mediante un sistema de categorías, consensuado por los investigadores, elaborado a partir de la propia observación, reducción y categorización de las respuestas aportadas por los estudiantes, para la posterior obtención de conclusiones.

\section{Resultados}

\subsection{Percepción del alumnado acerca de la tarea de evaluación "IOS"}

Los siguientes resultados se obtienen del análisis de los datos cuantitativos del Cuestionario Experiencia de Evaluación como Aprendizaje: percepción de los estudiantes EEA_(OSA), concretamente de los ítems P6_1, P6_2, P6_3, P6_4, P6_5, P6_6, P7_1, P7_2, P7_3, P7_4, P7_5, P8_1, P8_2, P8_3, P8_4, P8_5, P9_1, P9_2, P10_1, P10_2, P10_3, P10_4, P10_5, correspondientes a la percepción del alumnado acerca de la tarea de evaluación "IOS".

Se efectuó un análisis factorial exploratorio de los ítems mencionados, agrupados en las siguientes dimensiones: contexto real, tarea, recibir proalimentación, participar en la proalimentación y autoevaluación (ver cuadro 1), en el cual se determinó que estos se agrupaban en un solo factor, por lo que se estaría midiendo un solo constructo. La prueba de KMO $(0,918)$ arrojó un valor muy alto lo cual es una evidencia de la agrupación de los ítems. Además, la prueba de esfericidad de Barttler fue significativa $(0,000)$.

Como se puede observar en el cuadro 1, los ítems, cuyas cargas factoriales obtienen una puntuación menor de 0,6 (P7_05, P10_01 y P10_04), hacen referencia a la autoevaluación. Es por ello, que en el primer ítem señalado podría ser que el estudiante obtuvo la evaluación del docente, pero no del grupo, por lo tanto, de cara a una futura experiencia, proponemos dividirlo en tres opciones (alumno, docente y alumno y docente). En los otros dos casos, puede deberse a que esta modalidad de evaluación solo se empleó, de forma específica, en la valoración del producto final, pudiendo generar confusión. 
Cuadro 1. Carga factorial de los ítems correspondientes a las preguntas 6, 7, 8, 9 y 10

\begin{tabular}{|c|c|c|}
\hline Pregunta & ÍTEM & CARGA FACTORIAL \\
\hline \multirow{6}{*}{$\begin{array}{l}\text { P6. Realizar la tarea "Informe final de observación sistemática } \\
\text { aplicada en Educación Infantil" en un contexto educativo real, } \\
\text { me ha permitido: }\end{array}$} & P6_01 &, 715 \\
\hline & P6_02 &, 765 \\
\hline & P6_03 & ,727 \\
\hline & P6_O4 & ,756 \\
\hline & P6_05 & ,707 \\
\hline & P6_06 & ,681 \\
\hline \multirow{5}{*}{$\begin{array}{l}\text { P7. La tarea "Informe final de observación sistemática aplicada } \\
\text { en Educación Infantil"... }\end{array}$} & P7_O1 & ,779 \\
\hline & $\mathrm{P} 7 \_02$ & ,749 \\
\hline & P7_O3 & 619 \\
\hline & P7_O4 & ,805 \\
\hline & P7_O5 &, 568 \\
\hline \multirow{5}{*}{$\begin{array}{c}\text { P8. Reunirnos con el profesorado y recibir proalimentación } \\
\text { sobre las distintas prácticas/subtareas referentes al "Informe } \\
\text { final de observación sistemática aplicada en Educación Infantil”, } \\
\text { me ha permitido: }\end{array}$} & P8_01 & ,779 \\
\hline & P8_02 & ,776 \\
\hline & P8_03 & ,726 \\
\hline & P8_O4 & ,783 \\
\hline & P8_05 & ,706 \\
\hline \multirow{2}{*}{$\begin{array}{l}\text { P9. Participar activamente en el proceso de proalimentación, me } \\
\text { ha permitido: }\end{array}$} & P9_O1 & ,790 \\
\hline & P9_O2 & ,797 \\
\hline \multirow{5}{*}{$\begin{array}{l}\text { P10. Durante el desarrollo de la tarea "Informe final de } \\
\text { observación sistemática aplicada en Educación Infantil", has } \\
\text { tenido la posibilidad de evaluar tu trabajo, por ello consideramos } \\
\text { relevante que respondas el grado de acuerdo con las siguientes } \\
\text { afirmaciones. La autoevaluación... }\end{array}$} & P10_O1 &, 587 \\
\hline & P1O_02 & ,678 \\
\hline & P10_03 &, 724 \\
\hline & P10_O4 &, 582 \\
\hline & P10_05 & ,716 \\
\hline
\end{tabular}

Fuente: Elaboración propia.

En el gráfico de sedimentación (figura 7) se muestra la unidimensionalidad del constructo, la percepción del estudiante sobre la evaluación del aprendizaje en la asignatura Observación Sistemática y Análisis de Contextos. Por otra parte, la varianza total explicada fue de $53,48 \%$ cuyos ítems se agrupan en un solo factor.

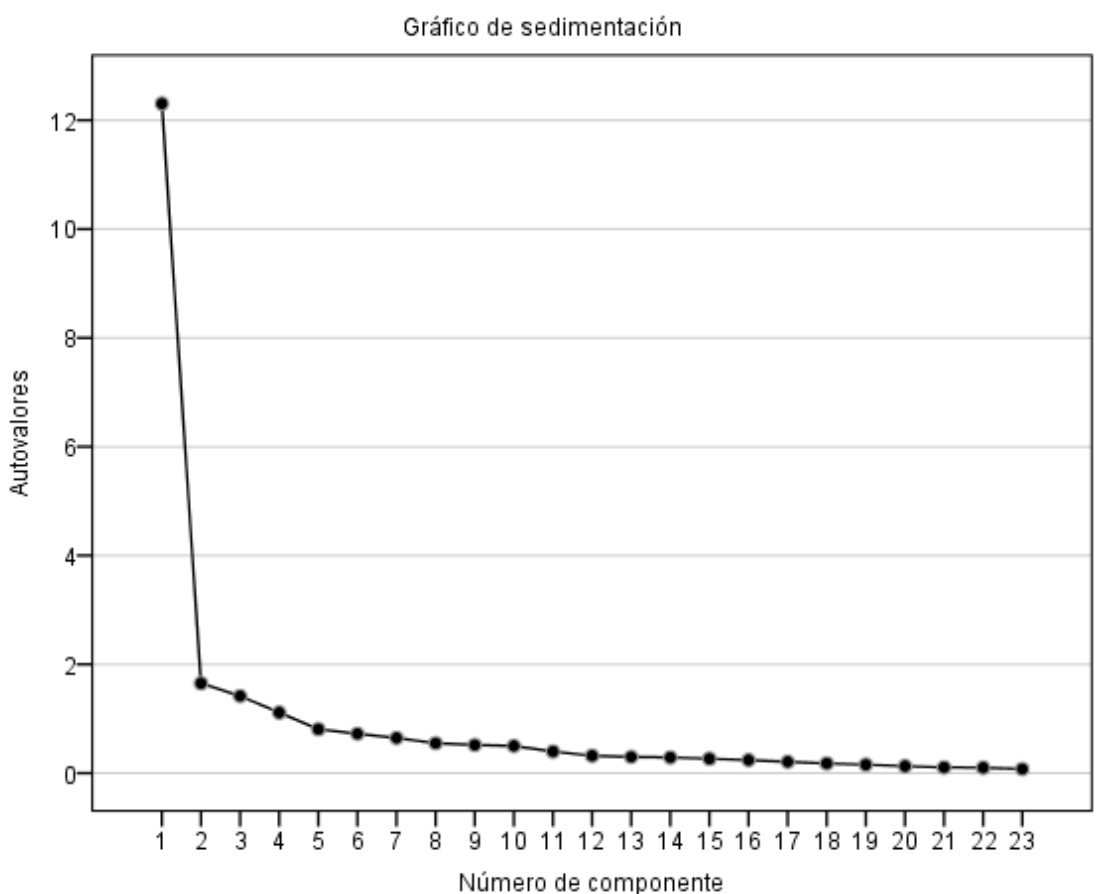

Figura 7. Gráfico de sedimentación para el cuestionario EEA_(OSA), considerando los 23 ítems correspondientes a las preguntas $6,7,8,9$ y 10

Fuente: Elaboración propia. 
Cuadro 2. Puntuaciones medias de las declaraciones referentes a la percepción del alumnado sobre la tarea de evaluación "IOS"

\begin{tabular}{|c|c|c|c|c|}
\hline & İTEM & DECLARACIÓN & MEDIA & D.T. \\
\hline \multirow{6}{*}{ 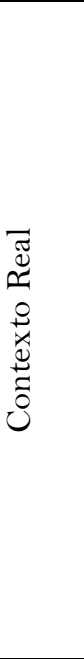 } & P6_1 & $\begin{array}{l}\text { Realizar la tarea en un contexto educativo real, me ha permitido } \\
\text { demostrar mi conocimiento de forma creativa para resolver } \\
\text { problemas de la vida real }\end{array}$ & 8,17 & 1,54 \\
\hline & P6_2 & $\begin{array}{l}\text { Realizar la tarea en un contexto educativo real, me ha permitido } \\
\text { aumentar mi motivación al realizar la tarea }\end{array}$ & 8,13 & 1,80 \\
\hline & P6_3 & $\begin{array}{l}\text { Realizar la tarea en un contexto educativo real, me ha permitido } \\
\text { comprender la observación sistemática como una técnica necesaria } \\
\text { para la reflexión sobre la práctica educativa }\end{array}$ & 9,08 & 1,25 \\
\hline & P6_4 & $\begin{array}{l}\text { Realizar la tarea en un contexto educativo real, me ha permitido } \\
\text { aplicar los instrumentos elaborados, proporcionándome el dominio, } \\
\text { las técnicas de observación y registro }\end{array}$ & 8,80 & 1,38 \\
\hline & P6_5 & $\begin{array}{l}\text { Realizar la tarea en un contexto educativo real, me ha permitido } \\
\text { elaborar conclusiones emergentes del análisis y la interpretación de } \\
\text { los datos adquiridos }\end{array}$ & 8,44 & 1,43 \\
\hline & P6_6 & $\begin{array}{l}\text { Realizar la tarea en un contexto educativo real, me ha permitido } \\
\text { ensayar, practicar, consultar fuentes, y lograr retroalimentación } \\
\text { para mejorar el producto final }\end{array}$ & 8,56 & 1,48 \\
\hline \multirow{5}{*}{ 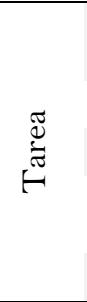 } & P7_1 & $\begin{array}{l}\text { La tarea se ha centrado en los procesos (el desarrollo y mejora } \\
\text { del resto de tareas) no solo en el producto }\end{array}$ & 8,38 & 1,81 \\
\hline & P7_2 & La tarea ha sido realista y retadora & 8,69 & 1,46 \\
\hline & P7_3 & La tarea ha sido compleja y de utilidad & 8,50 & 1,44 \\
\hline & $\mathrm{P} 7 \_4$ & $\begin{array}{l}\text { La tarea ha facilitado mi aprendizaje haciéndolo más útil y } \\
\text { significativo }\end{array}$ & 8,76 & 1,53 \\
\hline & $\mathrm{P} 7 \_5$ & La tarea ha sido evaluada por mi grupo y el docente & 8,63 & 1,65 \\
\hline \multirow{5}{*}{ 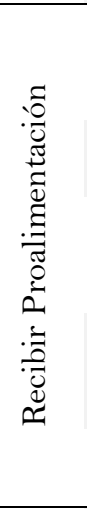 } & P8_1 & $\begin{array}{l}\text { Recibir proalimentación sobre las distintas prácticas/subtareas me } \\
\text { ha permitido conocer aquellos aspectos potenciales y débiles de mi } \\
\text { tarea }\end{array}$ & 8,44 & 1,46 \\
\hline & P8_2 & $\begin{array}{l}\text { Recibir proalimentación sobre las distintas prácticas/subtareas me } \\
\text { ha permitido conocer alternativas para mejorar mi tarea }\end{array}$ & 8,52 & 1,46 \\
\hline & P8_3 & $\begin{array}{l}\text { Recibir proalimentación sobre las distintas prácticas/subtareas me } \\
\text { ha permitido sentirme más seguro/a de cara al desarrollo de la } \\
\text { tarea final }\end{array}$ & 8,48 & 1,69 \\
\hline & P8_4 & $\begin{array}{l}\text { Recibir proalimentación sobre las distintas prácticas/subtareas me } \\
\text { ha permitido comprender mejor cómo realizar mis trabajos en esta } \\
\text { asignatura }\end{array}$ & 8,62 & 1,54 \\
\hline & P8_5 & $\begin{array}{l}\text { Recibir proalimentación sobre las distintas prácticas/subtareas me } \\
\text { ha permitido saber cómo hacerlo mejor en las siguientes tareas }\end{array}$ & 8,57 & 1,58 \\
\hline \multirow{2}{*}{ 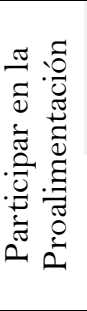 } & P9_1 & $\begin{array}{l}\text { Participar activamente en el proceso de proalimentación me ha } \\
\text { permitido aceptar las críticas a mi trabajo, entendiéndolas como } \\
\text { información útil, que ayudan a mejorar la calidad de la tarea y mi } \\
\text { aprendizaje }\end{array}$ & 8,85 & 1,28 \\
\hline & P9_2 & $\begin{array}{l}\text { Participar activamente en el proceso de proalimentación me ha } \\
\text { permitido comprender la evaluación como actividad que se } \\
\text { enriquece con el diálogo, sobre el objeto a evaluar, entre mis } \\
\text { compañeros y el profesorado }\end{array}$ & 8,77 & 1,26 \\
\hline \multirow{5}{*}{ 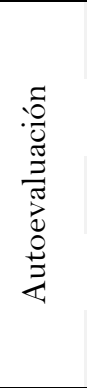 } & $\mathrm{P}_{1} 10_{-}$ & $\begin{array}{l}\text { La autoevaluación me ha ayudado a tener presente los criterios de } \\
\text { evaluación durante la elaboración de la tarea }\end{array}$ & 8,64 & 1,49 \\
\hline & $\begin{array}{l}\mathrm{P} 1 \mathrm{O}_{-} \\
2\end{array}$ & $\begin{array}{l}\text { La autoevaluación ha favorecido el desarrollo de mi capacidad de } \\
\text { autocrítica }\end{array}$ & 8,52 & 1,45 \\
\hline & $\begin{array}{l}\mathrm{P} 10_{-} \\
3\end{array}$ & La autoevaluación ha enriquecido mi aprendizaje & 8,81 & 1,37 \\
\hline & $\mathrm{P}_{4} 1 \mathrm{O}_{-}$ & $\begin{array}{l}\text { La autoevaluación ha hecho sentirme protagonista de mi propio } \\
\text { aprendizaje }\end{array}$ & 8,65 & 1,46 \\
\hline & $\begin{array}{l}\mathrm{P} 10_{-} \\
5\end{array}$ & $\begin{array}{l}\text { La autoevaluación me ha aportado información de utilidad para } \\
\text { realizar tareas similares en otros contextos }\end{array}$ & 8,58 & 1,44 \\
\hline
\end{tabular}

Fuente: Elaboración propia. 
En el cuadro 2 se pueden observar las medias y las desviaciones típicas de la percepción de los estudiantes en relación a la tarea de evaluación "IOS". Las declaraciones con puntuaciones medias más altas hacen referencia a: que la realización de la tarea en un contexto educativo real les ha permitido comprender la observación sistemática como una técnica necesaria para la reflexión sobre la práctica educativa (P6_3); que la participación en el proceso de proalimentación les ha permitido aceptar las críticas a su trabajo, entendiéndolas como información útil, que ayuda a mejorar la calidad de la tarea y su aprendizaje (P9_1) y que el tener la posibilidad de evaluar su trabajo ha enriquecido su aprendizaje (P10_3). Los datos reflejan cómo la declaración "la realización de la tarea en un contexto real ha incrementado mi motivación” (P6_2) presenta la menor puntuación media $(\bar{x}=8,13)$, seguida de las declaraciones "la realización de la tarea en un contexto real me ha permitido demostrar mis conocimientos de forma creativa para resolver problemas de la vida real" (P6_1) y "la tarea se ha centrado en los procesos (el desarrollo y mejora del resto de tareas) no solo en el producto" (P7_1).

La figura 8 muestra los porcentajes de las respuestas de los estudiantes, clasificadas en tres niveles de acuerdo (1-4 bajo, 5-7 medio y 8-10 alto) respecto a las declaraciones de la tarea de evaluación "IOS".

ロ1-4 $\square$ 5-7 $\square 8-10$

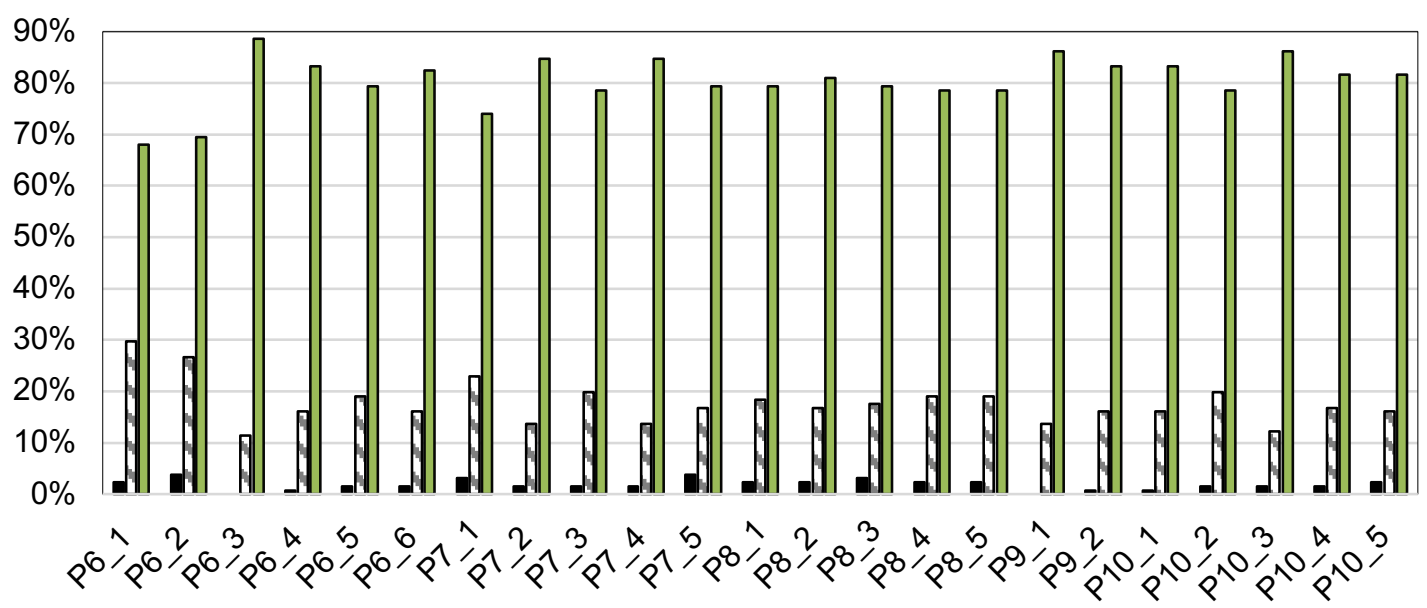

Figura 8. Porcentaje de respuestas clasificadas en niveles de acuerdo respecto a las declaraciones de la tarea de evaluación "IOS"

Fuente: Elaboración propia.

El $88,55 \%$ de los estudiantes muestra un nivel de acuerdo alto ante la afirmación "realizar la tarea en un contexto real, me ha permitido comprender la observación sistemática como una técnica necesaria para la reflexión sobre la práctica educativa" (P6_3). Además, el 86,26\% considera que la participación activa en el proceso de retroalimentación les ha permitido aceptar las críticas a su propio trabajo, entendiéndolas como información útil, que ayuda a mejorar la calidad de la tarea y su aprendizaje (P9_1). Igualmente, el $84,73 \%$ opina que la tarea ha facilitado su aprendizaje haciéndolo más útil y significativo (P7_4).

El $67,94 \%$ del estudiantado percibe que la realización de la tarea en un contexto real les ha permitido demostrar su conocimiento de forma creativa para resolver problemas de la vida real (P6_1), el 69,47\% indica que realizar la tarea en un contexto 
real ha incrementado su motivación (P6_2) y el 74,05\% reconoce que la tarea se ha centrado en los procesos (el desarrollo y mejora del resto de tareas) y no solo en el producto $\left(\mathrm{P} 7 \_1\right)$.

El cien por cien de los alumnos muestra un grado de acuerdo medio $(11,45 \%)$ y alto $(83,21 \%)$, en que la realización de la tarea en un contexto real les ha permitido comprender la observación sistemática como una técnicas necesaria para la reflexión sobre la práctica educativa (P6_3), del mismo modo, todos los estudiantes perciben, con un nivel de acuerdo medio (13,74\%) y alto $(86,26 \%)$, la proalimentación como una ayuda para la aceptación a las críticas de su propio trabajo, entendiéndolas como información útil, que ayudan a mejorar la calidad de la tarea y su aprendizaje (P9_1).

Cabe destacar que algunas declaraciones han sido valoradas en el nivel de acuerdo bajo, sin embargo, sus porcentajes están por debajo del cuatro por ciento, como, por ejemplo "La tarea ha sido evaluada por mi grupo y el docente" (P7_5) (3,82\%), "La tarea se ha centrado en los procesos (el desarrollo y mejora del resto de tareas) no solo en el producto" (P7_1) (3,05\%) y "Recibir proalimentación sobre las distintas prácticas/subtareas me ha permitido sentirme más seguro/a de cara al desarrollo de la tarea final” (P8_3) (3,05\%).

Menos del uno por ciento de los participantes $(0,76 \%)$ valoran con un nivel de acuerdo bajo que participar en el proceso de proalimentación les haya permitido comprender la evaluación como actividad que se enriquece con el diálogo, sobre el objeto a evaluar, entre los compañeros y el profesorado (P9_2). El mismo porcentaje de estudiantes $(0,76 \%)$ muestra un nivel de acuerdo bajo en que participar en un contexto real le haya permitido aplicar los instrumentos elaborados, proporcionándole el domino las técnicas de observación y registro (P6_4).

\subsection{Grado de satisfacción de los estudiantes con la evaluación de la segunda parte de la asignatura}

Los resultados mostrados a continuación proceden del análisis de los datos cuantitativos obtenidos de la pregunta $\mathrm{P}_{-} 12$ del cuestionario Experiencia de Evaluación como Aprendizaje: percepción de los estudiantes EEA_(OSA), estos hacen referencia al grado de satisfacción con la evaluación de la segunda parte de la asignatura OSA, de cada uno de los grupos (A, B y C) en los que se ha impartido la asignatura y al total de participantes.

El grupo A es el que presenta mayor puntuación media, en relación al grado de satisfacción con la evaluación $(\bar{x}=9,3)$, seguido del grupo $\mathrm{C}(\bar{x}=8,87)$. El grupo B es el que refleja menor puntuación $(\bar{x}=7,64)$. La media general, de todos los participantes, es superior a ocho $(\bar{x}=8,56)$.

La figura 9 muestra los porcentajes de las respuestas de los estudiantes, clasificadas en tres niveles (1-4 bajo, 5-7 medio y 8-10 alto) de acuerdo al grado de satisfacción con la evaluación de la segunda parte de la asignatura.

En la figura 9 podemos comprobar como en el grupo A, más de un noventa por ciento de los estudiantes (92\%) manifiestan un grado de satisfacción alto con la evaluación, a diferencia del grupo B, en el que tan solo más del cincuenta y cinco por ciento de los participantes $(56 \%)$ declaran dicho grado de satisfacción, aunque se debe señalar que el cuarenta por ciento de los estudiantes de este grupo presentan un grado de satisfacción 
medio. Más del ochenta por ciento de los participantes pertenecientes al grupo C indican un alto grado de satisfacción con la evaluación.

๑1-4 घ5-7 $\square 8-10$

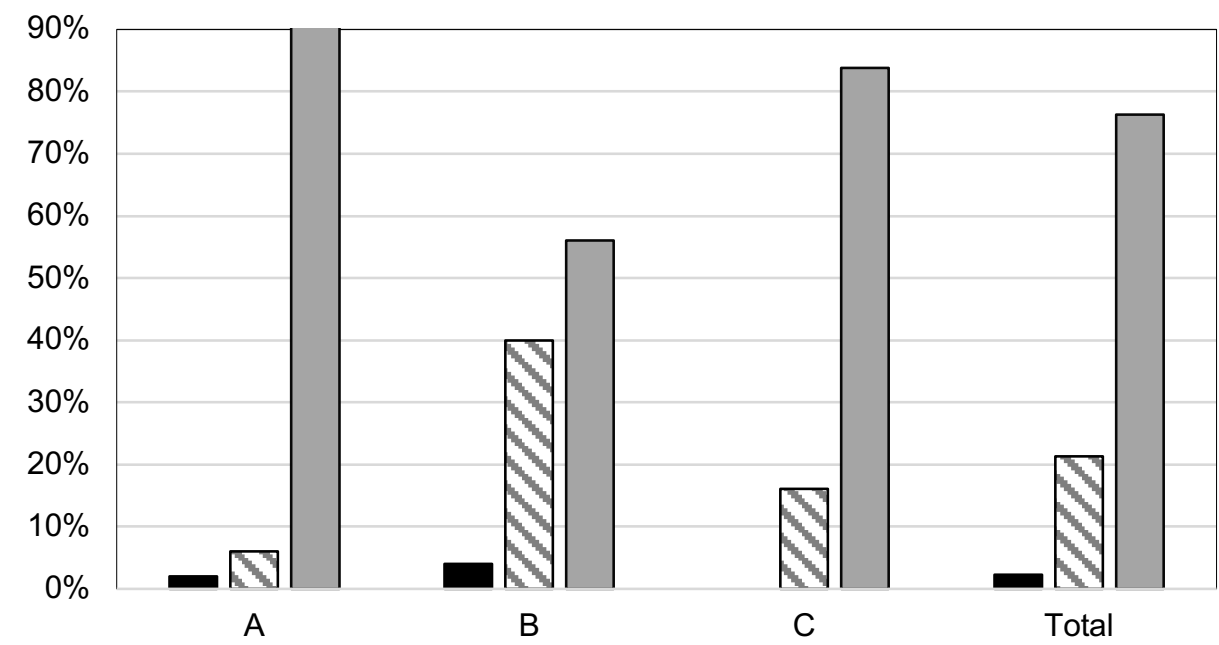

Figura 9. Grado de satisfacción de los estudiantes con la evaluación de la segunda parte de la asignatura, clasificada por grupos y total Fuente: Elaboración propia.

En el grupo $\mathrm{C}$ no hay estudiantes que manifiesten un grado de satisfacción bajo con la evaluación, menos de un cinco por ciento de los participantes de los grupos B (4\%) y A $(2 \%)$ indican tener un grado de satisfacción bajo en relación a la evaluación de la segunda parte de la asignatura.

En lo que respecta al porcentaje de respuestas totales, el $76,3 \%$ de los participantes presentan un grado de satisfacción alto, el 21,4\% presenta un grado de satisfacción medio y menos de un tres por ciento de los estudiantes presentan un grado de satisfacción bajo con la evaluación de la segunda parte de la asignatura.

\subsection{Aspectos positivos de la experiencia percibidos por los estudiantes}

En este apartado se exponen los resultados obtenidos del análisis cualitativo, extraído de las respuestas a las cuestiones abiertas P13, P14 y P15. Los aspectos positivos de la experiencia, indicados por los estudiantes, están relacionados con la evaluación como aprendizaje (F64), la experiencia en si misma (F26) y la interacción con el docente (F 11) (figura $10)$.

\subsubsection{Evaluación como aprendizaje}

$\mathrm{El}$ aspecto más destacado por los participantes, englobado en la categoría evaluación como aprendizaje, está enfocado en la realización de una tarea auténtica, algunos ejemplos son:

Es una buena idea poder realizar la tarea "informe final de la observación sistemática aplicada en educación infantil" con datos reales que nosotras mismas hemos podido recoger... (P13-S83)

Lo positivo es el hecho de trabajar una serie de prácticas que luego nos van a servir para realizar el informe final que las englobaría en su totalidad. (P13-S104)

Pues me ha permitido poner en práctica de manera real una tarea que realizaré en un futuro con lo cual se aprende de manera real. (P13-S34) 


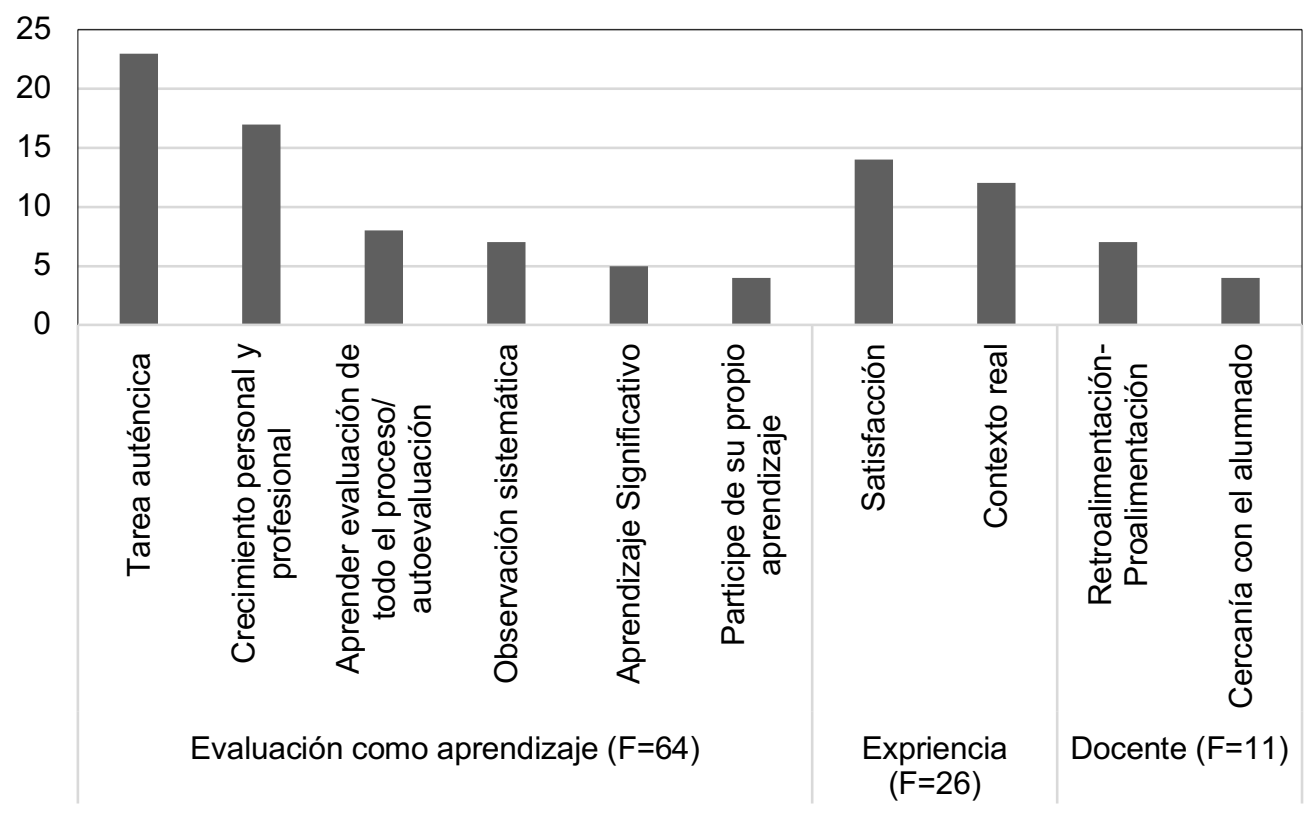

Figura 10. Sistema de categorías de los comentarios de los estudiantes sobre aspectos positivos de la experiencia Fuente: Elaboración propia.

Siguiendo con esta categoría, los estudiantes también señalan que la experiencia les ha aportado crecimiento personal y profesional, permitiéndoles desarrollar facetas personales y profesionales, algunas de las afirmaciones que se pueden presentar como ejemplos son:

Aprendes de forma diferente y se evalúa más el proceso que la calificación final, permitiendo mejorar y crecer como persona. (P13-S79)

... Nos ha ayudado a ser más críticos con nosotros mismos. (P13-S86)

Las clases me han parecido amenas. Y creo que esta forma de trabajar es muy positiva ya que nos permite conocer nuestros errores para poder corregirlos. (P 13-S33)

En esta categoría el estudiantado también destaca la importancia de aprender a autoevaluar todo el proceso de su propio aprendizaje expresando mediante frases como:

La evaluación de esta parte de la asignatura me ha parecido muy positiva, ya que valora el proceso de mejora del alumnado. (P13-S110)

... evaluarnos como nos gustaría a nosotros evaluar como futuros docentes ha servido de gran ayuda. (P13-S128)

No somos una nota, ni un conocimiento, y en esta prueba se puede evaluar correctamente. (P13-S101)

Otro aspecto resaltado por los estudiantes ha sido el aprendizaje de la observación sistemática, con oraciones como:

Considero que esta experiencia me ha servido para conocer los métodos de evaluación y sus instrumentos, aplicándolo en la práctica. (P13-S126)

He aprendido a utilizar los instrumentos y a analizarlos. (P13-S68)

...Hemos aprendido a utilizar los diversos instrumentos para la evaluación y su utilización. (P13-S86)

Los participantes también han destacado el aprendizaje significativo, con afirmaciones como: 
...Me ha ayudado a aprender de forma significativa $y$ he disfrutado a la vez que he aprendido. (P13-S17)

Ha sido una manera muy útil de evaluación en la que no hemos tenido que estudiar unos contenidos, sino aprenderlos. (P13-S106)

... Haciendo que nuestro aprendizaje sea más significativo. (P13-S83)

Para esta categoría los alumnos también han resaltado la posibilidad que se les da para poder ser partícipe de su propio aprendizaje, reflejándolo en frases como:

Este tipo de evaluación permite al alumnado ser partícipe de su aprendizaje. (P13-S23)

Haber podido participar en mi evaluación en las prácticas. (P13-S109)

Me ha gustado porque es una forma diferente de evaluar y te hace más partícipe. (P 13-S120)

\subsubsection{Experiencia}

Dentro de la categoría experiencia, los participantes han diferenciado entre respuestas orientadas a la satisfacción general con la experiencia, con argumentos como:

Me ha parecido enriquecedora y sobre todo he aprendido más en todo este proyecto que en muchas asignaturas... (P13-S128)

En general, estoy muy satisfecha con la $2^{a}$ parte de la asignatura. (P13-S43)

Me ha parecido muy enriquecedora. (P13-S92)

y respuestas enfocadas a desarrollar la experiencia en un contexto real, con expresiones como:

Me ha gustado mucho la experiencia de poder observar una clase de educación infantil. (P13S76)

...El simple hecho de observar a los niños me hace sentir que esta mitad de la asignatura me ha servido para algo y para aprender más, no como otras que solo sirven para reflejar una nota. (P13-S81)

Nos acerca al mundo educativo. (P13-S129)

\subsubsection{Docentes}

Los estudiantes también han destacado aspectos positivos relacionados con los docentes, haciendo alusión a la retroalimentación y proalimentación:

Me ha parecido bien que el profesor nos fuera guiando en el proceso, aportándonos cómo corregir los errores. (P13-S61)

Me ha parecido que la asignatura se ha dado adecuadamente haciendo en todo momento partícipe al alumnado, y también haciéndoles ver cuáles eran sus errores y cuáles sus puntos fuertes del trabajo, pero siempre de forma conjunta. (P13-S45)

Retroalimentación con la docente. Críticas constructivas a la hora de realizar el informe final. (P13-S72)

Por último, como aspecto positivo, se ha señalado la cercanía del profesorado con los estudiantes, a modo de ejemplo podemos destacar la siguiente afirmación:

Contacto con alumnos/as. (P13-S111)

La disposición de la profesora... y la cercanía nos ha ayudado muchísimo positivamente. (P13-S105)

\subsection{Aspectos negativos de la experiencia percibidos por los estudiantes}

En la figura 11, expuesta a continuación, se presenta el análisis de las respuestas de los estudiantes en relación a los aspectos negativos percibidos en la experiencia. Las 
categorías emergentes del análisis son: la falta de tiempo (F33), aspectos relacionados con el diseño y planificación de la asignatura (F24), ninguno (F8) y la falta de feedbak o retroalimentación $(\mathrm{F}=6)$.

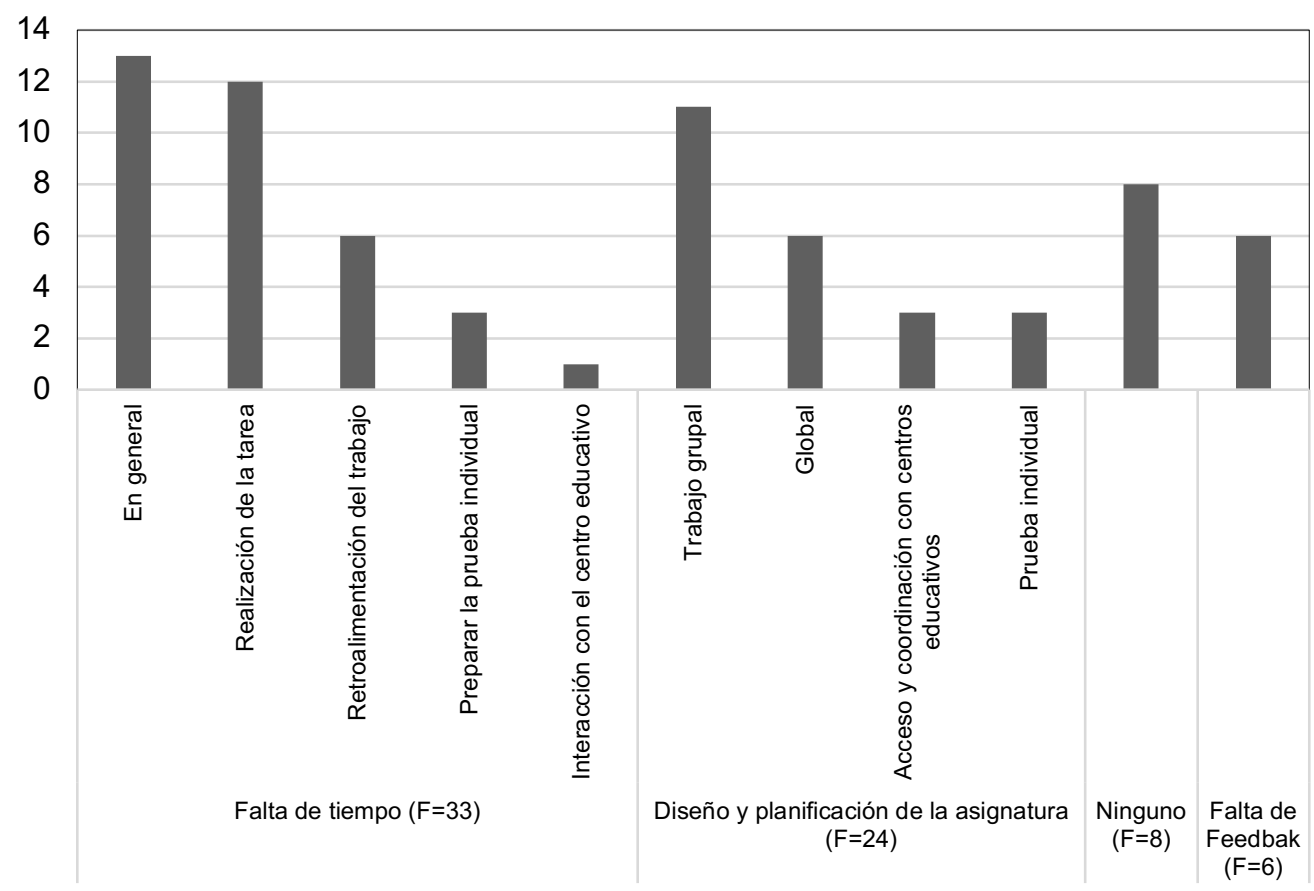

Figura 11. Sistema de categorías de los comentarios de los estudiantes sobre aspectos negativos de la experiencia Fuente: Elaboración propia.

\subsubsection{Falta de tiempo}

Dentro de la categoría falta de tiempo se destaca la subcategoría que hacen alusión a dicha falta de tiempo de manera general, con afirmaciones como:

Con respecto al aprendizaje, formación y contenido no tendría nada negativo que destacar. Si tuviera algo menos positivo que decir me decantaría por el tiempo. (P14-S55)

No hemos tenido tiempo suficiente para hacerlo todo de forma correcta y aprender todo lo que queríamos. (P14-S61)

Quizás sea una práctica para hacer con más tiempo, ya que pienso que es fundamental para la actividad docente. (P14-S80)

En la misma categoría los participantes han manifestado falta de tiempo para la realización de la tarea, con expresiones como:

Al tener poco tiempo de clases, por diversas circunstancias, se nos ha acumulado el trabajo de la asignatura siendo un poco estresante y, la realización de la observación era bastante compleja para el tiempo del que disponíamos. ( $\mathrm{P} 14-\mathrm{S} 17)$

Creo que hemos tenido poco tiempo para la realización de las prácticas, muchas de nosotras venimos de Bachillerato y es la primera vez que nos enfrentamos a algo como esto. (P14-S82)

Si tuviera que recalcar algo negativo sería la falta de tiempo a la hora de realizar la observación en el aula, ya que, si hubiésemos podido observar durante un periodo largo de tiempo, hubiésemos podido ver unos resultados más exhaustivos. (P14-S20)

Los sujetos afirman la necesidad de más tiempo para una correcta retroalimentación del trabajo, lo manifiestan con frases como: 
Muy poco tiempo para la corrección por parte de la profesora para mejorar el trabajo. (P14S129)

... No siempre daba tiempo a que el profesor nos resolviese bien las dudas a todas. (P14-S82)

Falta de tiempo para la corrección de los proyectos en el aula. (P14-S119)

El estudiantado destaca la falta de tiempo para una adecuada preparación para la prueba individual (DIEVED), con comentarios como:

... Y lo mismo podría decir de la prueba, que tuvimos menos tiempo del esperado... (P14$\mathrm{S} 55)$

Creo que es necesario que en clase se realicen más practicas comunes similares a la práctica D para así tener una mejor idea para la prueba final. (P14-S4)

En mi opinión se debería haber hecho más prácticas como el informe final. (P14-S86)

Los estudiantes también manifestaron falta de tiempo en la interacción con los centros educativos, con la siguiente frase:

El tiempo que hemos tenido para llevar a la práctica en la Escuela Infantil ha sido escaso. (P14-S85)

\subsubsection{Diseño y planificación}

En lo referente a los comentarios negativos relacionados con el diseño y planificación, algunos estudiantes han expresado encontrar inconvenientes relacionados con el trabajo en grupo, expresando ideas como:

En cuanto a lo negativo hay bastante poco por no decir nada, quizás el tema de los grupos de trabajos que a veces hay sus más y sus menos y no siempre todos trabajan por igual, pero en general todo bien. (P14-S128)

Cuando se realizan actividades en grupo cabe la posibilidad de que algún o algunos de los integrantes deje de hacer las cosas. (P 14-S60)

El trabajo en grupo es difícil a la hora de recolectar la información y ponerla en común ya que todos no trabajan igual. (P14-S1)

Otros estudiantes han destacado aspectos generales relacionados con el diseño y planificación de la asignatura global, algunos ejemplos son:

No tengo nada negativo que aportar de la experiencia, si acaso, pienso que la segunda parte de la asignatura debería tener más clases que la primera parte. (P14-S62)

Puede ser pesado estar continuamente evaluando cada paso que damos. (P14-S51)

Lo único que no me ha parecido muy acertado es el abanico tan amplio que hemos tenido para la realización de la observación, pienso que deberíamos haber tenido unos temas marcados desde el inicio, ya que hubiera facilitado aún más el inicio de las actividades. Esa es la única apreciación negativa que tengo de la asignatura. (P14-S43)

Algunos sujetos expresaron dificultades a la hora de acceder y coordinarse con los centros educativos en los que se desarrolla parte de la tarea, algunas frases de ejemplo son:

Ha sido difícil encontrar un centro el cual nos permitiera ir las horas que necesitábamos para no entorpecer las clases. (P14-S60)

y que nos tengamos que buscar nosotros los colegios y son dificiles de encontrar. (P14-S98)

No poder asistir al aula en los horarios que necesitábamos. (P 14-S13)

También señalan como negativos aspectos relacionados con la tarea de evaluación "DIEVED”, con expresiones como: 
En mi caso, y a pesar de poder tener los apuntes en el examen, tuve un bloqueo al principio que quizás repercuta negativamente en mi nota. (P14-S110)

Haber realizado una prueba individual, cuando pienso que la práctica ha estado más trabajada que esa prueba última. (P14-S109)

\subsubsection{Ningun aspecto negativo}

Algunos de los comentarios del estudiantado que no percibe la existencia de aspectos negativos son:

No considero ningún aspecto negativo en esta experiencia. (P14-S1 18)

No considero que exista ningún aspecto negativo de esta experiencia. Por poner uno, creo que podríamos haber hecho algún informe más en clase para mejorar nuestro conocimiento, pero tampoco lo veo necesario. (P14-S126)

No considero que tenga que objetar nada con respecto a lo que se me solicita en esta pregunta, debido a que, como he contestado anteriormente, en mi opinión, todo lo desarrollado en el aula ha sido satisfactorio y gratificante. (P14-S88)

\subsubsection{Falta de retroalimentación}

Algunos estudiantes expresan la falta de retroalimentación de la experiencia, como, por ejemplo:

No saber a veces exactamente qué poner en cada parte del informe. (P14-S104)

Siento que deberíamos de haber mirado más las prácticas anteriores para ver si íbamos bien encaminados. (P14-S120)

Lo único que no me ha gustado es que no he sabido si estaba todo bien o en qué me he equivocado. (P14-S103)

\subsection{Propuestas de mejora aportadas por los estudiantes}

En la figura 12, se exponen los resultados obtenido tras el análisis de los comentarios y observaciones de los participantes acerca del proceso de evaluación de la asignatura y la experiencia en general, con el fin de mejorar la práctica educativa.

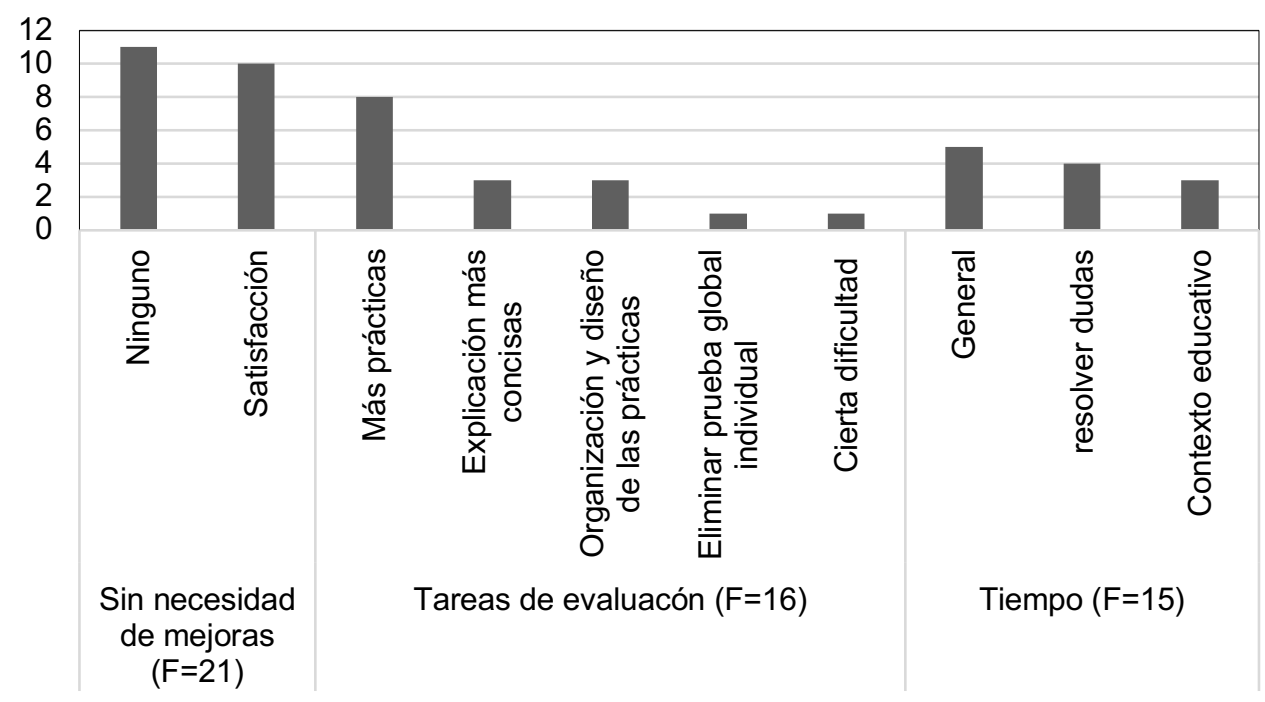

Figura 12. Sistema de categorías de los comentarios de los estudiantes sobre propuestas de mejora

Fuente: Elaboración propia. 


\subsubsection{Sin necesidad de mejora}

Ciertos comentarios realizados por los participantes hacen alusión a que no consideran necesario realizar cambios en la evaluación del aprendizaje, algunos ejemplos son:

Bajo mi punto de vista no creo que haya que mejorar nada con respecto a esta parte de la asignatura. (P15-S9)

Estoy de acuerdo con la evaluación del aprendizaje que se sigue en esta asignatura, porque permite al alumnado un aprendizaje centrado más en el proceso que en los resultados. (P15S23)

Nada que mejorar. (P15-S57)

Algunos estudiantes reflejan estar satisfechos con la evaluación del aprendizaje en sus respuestas, como se puede comprobar en las siguientes afirmaciones:

... me ha parecido muy provechosa la experiencia para saber en qué aspectos fijarnos y atender para evaluar todo el proceso de aprendizaje de nuestro alumnado. (P15-S40)

Me ha parecido interesante y útil dicha forma para realizar las prácticas de la asignatura ya que normalmente siempre tratamos con prácticas más teóricas que dinámicas, y al experimentar tal forma nos produce más satisfacción de ir a la escuela para poner en práctica nuestro trabajo. (P15-S52)

Me parece una evaluación muy útil y gratificante. (P15-S59)

\subsubsection{Tareas de evaluación}

Los estudiantes proponen tener más prácticas y actividades relacionadas con las tareas de evaluación, expresando frases como:

Hacer en clase algún informe más. (P15-S126)

No solo centrarnos en una sola práctica. (P15-S117)

Creo que deberíamos de haber hecho más prácticas sobre otros temas debido a que hubiéramos tenido más amplitud para evaluar. (P 15-S45)

Los participantes indican que las tareas de evaluación deben presentarse con explicaciones más concisas, mediante la redacción de las siguientes frases:

Mayor explicación colectiva de lo que se pide en la práctica. (P15-S104)

Me gustaría que los ejercicios se explicasen con mayor detalle ya que alguna vez mi grupo y yo hemos tenido dudas en realizar alguna actividad e incluso el trabajo final. (P15-S28)

Mejorar la claridad y explicaciones de las prácticas. (P15-S37)

Un estudiante propone eliminar la tarea de evaluación DIEVED:

Eliminar la prueba individual y tener más en cuenta la práctica. (P15-S37)

y otro estudiante señala la dificultad a la hora de realizar algunos aspectos de la tarea:

Ha sido cómodo a la hora de tener una guía en la que apoyarse para realizar las prácticas, pero había puntos de la guía que eran un poco complicados de realizar. (P15-S37)

\subsubsection{Tiempo}

Los estudiantes han sugerido disponer de más tiempo para el desempeño de la tarea. Algunos han propuesto disponer de más tiempo de manera general, algunos ejemplos son:

El único "pero" que le pongo a la evaluación es que me hubiera gustado tener más tiempo para poder aprender más. (P15-S66)

Contar con más tiempo de manera que nos podamos centrar mejor en todo este proceso. (P15S34) 
Quizás a esta segunda parte lo único que le añadiría sería más tiempo. Porque hemos tenido lo suficiente, pero sé que con más hubiésemos aprendido mucho más. (P15-S128)

Otros han remarcado la necesidad de más tiempo para la resolución de dudas:

Lo programaría de forma que no vayamos tan justos de tiempo y el profesor pueda dedicar un poco más de tiempo a cada grupo para la corrección de los grupos. (P15-S100)

Solo creo que hace falta algo más de tiempo (una o dos sesiones) para aclarar mejor las dudas que se puedan tener y mejorar la evaluación. (P15-S129)

Tener un control de la revisión porque nos faltaba tiempo para que se lo mirases a todos los grupos. (P15-S60)

Los participantes proponen disponer de más tiempo de contacto con los centros educativos en los que se realiza la observación sistemática, expresando frases como:

lo único que creo que se podría modificar, sería la falta de tiempo a la hora de realizar la observación en el aula de infantil. (P15-S20)

Estaría bien que se dejara más tiempo para poder ir a observar al aula ya que la mayoría de los grupos solo hemos podido ir una vez. (P15-S116)

Para realizar una buena observación, pienso que se debería de ampliar el tiempo de estancia en las aulas, con un día no es suficiente para desarrollar la observación plenamente. (P15$\mathrm{S} 40$ )

También plantean comentarios en los que sugieren que para mejorar la evaluación del aprendizaje sería necesario tener más tiempo para preparar el informe final de la tarea "IOS", como, por ejemplo:

Como he dicho anteriormente, creo que ha faltado tiempo. Por ejemplo, en el tema de los análisis, que en general, andábamos un poco más perdidos. (P15-S87)

Pienso que se debería ampliar un poco más el plazo para la realización del informe final. (P15-S118)

Poco tiempo para terminar la práctica. (P15-S53)

\section{Discusión y conclusiones}

El objetivo de este estudio era conocer la percepción de los estudiantes universitarios acerca del proceso de evaluación de la segunda parte de la asignatura Observación Sistemática y Análisis de Contextos.

Dando respuesta al primero de nuestros objetivos específicos, los estudiantes perciben que la tarea de evaluación "Elaboración de un Informe de observación sistemática aplicada en un centro/contexto de Educación Infantil”, les ha permitido aumentar la motivación y comprender la observación sistemática como una técnica necesaria para la reflexión sobre la práctica educativa. Los resultados evidencian que cumple todas las características de las tareas auténticas especificadas por Rodríguez-Gómez e Ibarra-Sáiz (2011), siendo aludidas en mayor medida por el alumnado las siguientes: permite la construcción del conocimiento y demostración en contextos reales, es útil y significativa, realista y retadora, compleja y de utilidad, centrada en el proceso no solo en los resultados y facilita el empoderamiento sobre su propio aprendizaje y evaluación. Así mismo, los estudiantes han percibido que participar activamente en el proceso de proalimentación a lo largo de la tarea, les ha proporcionado mayores beneficios que únicamente recibir los comentarios que provienen del profesorado. Esto es debido a que el nivel de autorregulación en este último caso, predominante hoy en día en la Educación Superior (Bearman et al., 2016), es casi 
inexistente (García-Jiménez et al., 2013). Se destaca entonces, la necesidad de pasar de la retroalimentación a la proalimentación, a través de estrategias de evaluación democráticas (Murillo e Hidalgo, 2016), promoviendo el diálogo compartido entre docente y estudiante para que éste utilice toda la información y decida cómo regular su aprendizaje (GarcíaJiménez et al., 2013).

En relación al segundo objetivo específico, los datos confirman que gran parte de los estudiantes presentan un grado de satisfacción alto con la evaluación de la segunda parte de la asignatura. No obstante, se han encontrado algunas diferencias en función del grupo al que pertenecen los estudiantes, obteniendo mayor nivel de satisfacción los estudiantes pertenecientes a los grupos A y C, frente a los del grupo B. Esto puede deberse al papel del docente en el proceso de evaluación en función de su experiencia, conocimiento sobre el tema, etc. ya que, de los dos docentes implicados en la experiencia, uno de ellos impartía los grupos A y C y el otro el B. Esto podría indicar que es necesaria una capacitación formal de los docentes para profesionalizarse en evaluación (Norton, Floyd y Norton, 2019) que les permita implementar estrategias participativas en sus asignaturas de una manera competente (Quesada-Serra, Rodríguez-Gómez e Ibarra-Sáiz, 2016), rompiendo con las prácticas predominantes en evaluación a través de innovaciones en los diseños de evaluación (Quesada-Serra, Rodríguez-Gómez e Ibarra-Sáiz, 2017).

En cuanto al tercer objetivo específico de nuestra investigación, como aspectos positivos de la experiencia, se ha destacado poder realizar una tarea auténtica en un contexto real, permitiéndoles dicha práctica desarrollar aspectos útiles para su desarrollo personal y profesional, siendo esta enriquecedora y satisfactoria. Lo cual ha permitido a los estudiantes "concebir su verdadero aporte a la solución de tareas mediante el disfrute" (Hortigüela, Pérez-Pueyo y González-Calvo, 2019, p. 23), empoderándose a través del proceso (Rodríguez-Gómez e Ibarra-Sáiz, 2015). Los aspectos negativos y propuestas de mejora se han enfocado en la falta de tiempo para la realización de la tarea y para la retroalimentación del trabajo, convirtiéndose este recurso limitado en una de las principales dificultades a la que se enfrentan, tanto docentes como estudiantes, en este tipo de prácticas evaluativas respecto a la carga de trabajo que conllevan (Henderson, Ryan y Phillips, 2019). Para garantizar la sostenibilidad de este planteamiento de evaluación es necesario "realizar un ejercicio de adecuación y proporcionalidad en función de su realidad: número de alumnos por aula, duración de la asignatura, tipo y número de tareas demandadas..." (Hortigüela, et al., 2019, p. 22).

El estudio realizado ha evidenciado que los docentes y estudiantes de esta asignatura están alineados en cuanto a la autenticidad de la tarea de evaluación, pudiendo no ser así al entrar en juego la subjetividad del concepto y su vinculación con las percepciones entre docentes y alumnos (Vallejo y Molina, 2014). Esta ha permitido a los estudiantes aplicar aquellos aspectos, conceptuales actitudinales y procedimentales, a trabajar en la materia, en un contexto real, lo que da un sentido pragmático al trabajo que realizan, permitiéndoles desarrollar competencias útiles para su vida personal y profesional (Rodríguez-Gómez e Ibarra-Sáiz, 2015). Por todo ello, podemos decir que a pesar del tiempo y esfuerzo que puede requerir el diseño de tareas de evaluación auténticas los beneficios en términos de mejora de aprendizaje de los estudiantes las convierte en imprescindibles (Brown, 2015). 


\section{Referencias}

Ahumada A. P. (2005). La evaluación auténtica: un sistema para la obtención de evidencias y vivencias de los aprendizajes. Perspectiva Educacional. Formación de Profesores, 45, 11-24.

Bearman, M., Dawson, P., Boud, D., Bennett, S., Hall, M. y Molloy, E. (2016). Support for assessment practice: Developing the assessment design decisions framework. Teaching in Higher Education, 21(5), 545-556. https://doi.org/10.1080/13562517.2016.1160217

Bilbao Martínez, A. y Villa Sánchez, A. (2019). Avances y limitaciones en la evaluación del aprendizaje a partir del proceso de convergencia. Visión docente y discente en los grados de Educación Infantil y Primaria. Educación XX1, 22(1), 45-69. https://doi.org/10.5944/educXX1.19976

Boud, D. (2000). Sustainable assessment: rethinking assessment for the learning society. Studies in Continuing Education, 22(2), 151-167. https://doi.org/10.1080/713695728

Boud, D. (2010). Assessment 2020: Seven propositions for assessment reform in higher education. Sydney: Australian Learning and Teaching Council.

Brown, S. (2015). La evaluación auténtica: el uso de la evaluación para ayudar a los estudiantes a aprender. RELIEVE, 21(2), art. M4. https://doi.org/10.7203/relieve.21.2.7674

Buendía, L., Colás, P. y Hernández, L. (1997). Métodos de Investigación en psicopedagogía. Madrid: McGraw-Hill.

Carless, D., Joughin, G. y Mok, M. M. C. (2006). Learning-Oriented Assessment: Principles and Practice. Assessment y Evaluation in Higher Education, 31(4), 395-398. https://doi.org/10.1080/02602930600679043

Cubero-Ibáñez, J. (2017). Desarrollo de competencias transversales en Educación Superior a través de la evaluación participativa y simulación en entornos virtuales de aprendizaje. Tesis doctoral inédita. Universidad de Cádiz, Cádiz.

García-Jiménez, E., Ibarra-Sáiz, M. S. y Rodríguez-Gómez, G. (2013). Guía PROALeval_DI. Diseño e implementación de la retroalimentación y proalimentación en la universidad. Cádiz: EVALfor Grupo de Investigación. https://doi.org/0.13140/RG.2.1.4513.1607

Gómez Ruíz, M. y Quesada-Serra, V. (2017). Coevaluación o Evaluación Compartida en el Contexto Universitario: La Percepción del Alumnado de Primer Curso. Revista Iberoamericana de Evaluación Educativa, 10(2), 9-30. https://doi.org/10.15366/riee2017.10.2.001

Henderson, M., Ryan, T. y Phillips, M. (2019). The challenges of feedback in higher education. Assessment y Evaluation in Higher Education, 44(8), 1237-1252. https://doi.org/10.1080/02602938.2019.1599815

Herrington, A. y Herrington, J. (2006). Authentic learning environments in higher education. Hershey, PA: Information Science Publishing.

Hortigüela, D., Pérez-Pueyo, Á. y González-Calvo, G. (2019). Pero... ¿A qué nos referimos realmente con la evaluación formativa y compartida?: Confusiones habituales y reflexiones prácticas. Revista Iberoamericana de Evaluación Educativa, 12(1), 13-27. https://doi.org/10.15366/riee2019.12.1.001

Ibarra-Sáiz, M. S. y Rodríguez-Gómez, G. (2015). Conceptos básicos en Evaluación como aprendizaje y empoderamiento en la Educación Superior. Cádiz: EVALfor - Grupo de Investigación. https://doi.org/10.13140/RG.2.1.5070.5686 
Ibarra-Sáiz, M. S., Rodríguez-Gómez, G. y García-Jiménez, E. (2016). Tareas de calidad: Caracterización. Cádiz: EVALfor - Grupo de Investigación. Recuperado de https://www.youtube. com/channel/UCjp_eJyqxOKtVQ68f4QlH-Q/playlists

Ibarra-Sáiz, M. S. y Rodríguez-Gómez, G. (2014). Modalidades participativas de evaluación: Un análisis de la percepción del profesorado y de los estudiantes universitarios. Revista de Investigación Educativa, 32(2), 339-361. https://doi.org/10.6018/rie.32.2.172941

Lukas, J.F., Santiago, K., Lizasoain, L., y Etxeberria, J. (2017). Percepciones del alumnado universitario sobre la evaluación. Bordón. Revista de pedagogía, 69(1), 103-122.

Murillo, F. J. e Hidalgo, N. (2016). Evaluación democrática y para la democracia. Revista Iberoamericana de Evaluación Educativa, 9(1), 5-7.

Norton, L., Floyd, S. y Norton, B. (2019). Lecturers' views of assessment design, marking and feedback in higher education: a case for professionalisation? Assessmenty Evaluation in Higher Education, 44(8), 1209-1221. https://doi.org/10.1080/02602938.2019.1592110

Panadero, E., Fraile, J., Fernández-Ruiz, J., Castilla-Estévez, D. y Ruiz, M. A. (2018). Spanish university assessment practices: Examination tradition with diversity by faculty. Assessment y Evaluation in Higher Education, 44(3), 1-19. https://doi.org/10.1080/02602938.2018.1512553

Quesada-Serra, V., Gómez Ruiz, M. A., Gallego Noche, B. y Cubero-Ibáñez, J. (2019). Should I use co-assessment in higher education? Pros and cons from teachers and students' perspectives, Assessment y Evaluation in Higher Education, 44(7), 987-1002. https://doi.org/10.1080/02602938.2018.1531970

Quesada-Serra, V., Rodríguez-Gómez, G. e Ibarra-Sáiz, M. S. (2016). What are we missing? Spanish lecturers' perceptions of their assessment practices. Innovations in Education and Teaching International, 53(1), 48-59.

Quesada-Serra, V., Rodríguez-Gómez, G. e Ibarra-Sáiz, M. S. (2017). Planificación e innovación de la evaluación en educación superior: la perspectiva del profesorado. Revista de Investigación Educativa, 35(1), 53-70.

Rodríguez-Gómez, G. e Ibarra-Sáiz, M. S. (Eds.). (2011). e-Evaluación orientada al e-Aprendizaje estratégico en Educación Superior. Madrid: Narcea.

Rodríguez-Gómez, G. e Ibarra-Sáiz, M. S. (2015). Assessment as learning and empowerment: beyond sustainable learning in Higher Education. En M. Peris-Ortiz y J. M. Merigó Lindahl (Eds.), Sustainable learning in higher education, innovation, technology, and knowledge management. (pp. 1-20). Londres: Springer-Verlag. https://doi.org/10.1007/978-3-319-10804-9_1

Rodríguez-Gómez, G., Ibarra-Sáiz, M. S. y Cubero-Ibáñez, J. (2018). Competencias básicas relacionadas con la evaluación. Un estudio sobre la percepción de los estudiantes universitarios. Educación XX1, 21(1), 181-208. https://doi.org/10.5944/educXX1.14457

Vallejo Ruiz, M. y Molina Saorín J. (2014). La evaluación auténtica de los procesos educativos. Revista Iberoamericana de Educación, 64, 11-25. https://doi.org/https://doi.org/10.35362/rie640403

Vu, T. y Dall'Alba, G. (2014). Authentic assessment for student learning: an ontological conceptualisation. Educational Philosophy and Theory 46(7) 778-791.

https://doi.org/10.1080/00131857.2013.795110 


\section{ANEXO I}

\section{PRÁCTICA D}

\section{INFORME FINAL DE OBSERVACIÓN SISTEMÁTICA APLICADA EN UN CENTRO/CONTEXTO DE EDUCACIÓN INFANTIL}

Una vez hemos realizado nuestra investigación, debemos presentarla en un informe. Debemos tener en cuenta que el informe no solo refleja los resultados obtenidos, sino que también debe contar con una justificación teórica de la temática investigada, una descripción de la metodología usada y las conclusiones a las que llegamos tras el análisis de resultados. Los apartados y los contenidos que debe tener nuestro informe son los siguientes:

1. Título: debe resumir en una frase nuestra investigación.

2. Revisión de la literatura y planteamiento de nuestro problema, incluyendo la pregunta de investigación, a la que se quiere dar respuesta con nuestra observación: Justificaremos el sentido o la importancia de nuestro problema, concretando la pregunta de investigación a la que queremos dar respuesta, e incluiremos la revisión bibliográfica tanto de teorías como de investigaciones parecidas en relación con nuestra temática.

\section{Metodología}

a. Participantes: describiremos el proceso de selección de los informantes (sujetos a los que observamos) y las situaciones o contexto a observar.

b. Instrumentos: presentaremos el o los instrumentos de recogida de datos que hemos diseñado. Para ello los describiremos en profundidad, así como haremos referencia a sus características, incluyendo los originales.

c. Procedimiento de recogida de información: describiremos el proceso que hemos seguido para la recogida de información. En esta parte podemos incluir el tipo de observación realizada tomando en cuenta a los/as observadores/as. Se incluirán en ANEXOS los instrumentos cumplimentados por cada observador/a, identificando cada uno de ellos (Observadora 1: Nombre, Observadora 2: Nombre, etc.)

d. Análisis de los datos: haremos referencia al tipo de análisis que hemos realizado con cada tipo de dato.

4. Resultados: presentaremos los resultados obtenidos tanto de los datos cuantitativos como cualitativos. Para la presentación de los datos cuantitativos haremos uso de las tablas y las gráficas y para los datos cualitativos agruparemos la información atendiendo al sistema de categorías que hemos creado. 
5. Conclusiones: debe aparecer un análisis de los hallazgos tras la presentación de los resultados (dando respuesta a la pregunta inicial que nos planteábamos). Debemos conectar los resultados con las aportaciones teóricas de los autores que hemos referenciado en nuestro marco teórico u otros. En la última parte de las conclusiones podemos incluir aspectos a mejorar de nuestra investigación o prospectiva de la misma.

6. Referencias bibliográficas: deben seguir las normas APA $6^{\text {a }}$ Edición.

7. Anexos: con los datos originales (Anexo I: Instrumentos cumplimentados por los/as observadores/as), se pueden añadir otros ANEXOS que creáis convenientes.

\section{Producto}

Se entregará un único documento con los apartados comentados con formato PDF, DOCX o DOC que se subirá al Campus Virtual como máximo el día 5 de junio.

Sólo el coordinador o coordinadora del grupo debe entregarlo y se identificarán tanto el nombre del grupo como los componentes del grupo en el cuerpo del documento.

Se redactará con letra Arial 11 con interlineado sencillo.

\section{Criterios, instrumento y modalidad de evaluación}

Para la autoevaluación grupal del informe haremos uso de la siguiente escala de valoración (siendo 1 el valor mínimo y 5 el máximo):

\begin{tabular}{|c|c|c|c|c|c|}
\hline \multirow{2}{*}{ DIMENSIONES } & \multicolumn{5}{|c|}{ VALORACIÓN } \\
\hline & 1 & 2 & 3 & 4 & 5 \\
\hline \multicolumn{6}{|l|}{ Aspectos generales } \\
\hline \multicolumn{6}{|l|}{ Ortografía cuidada y expresión clara y correcta } \\
\hline \multicolumn{6}{|l|}{ Cumplimiento de las normas (formato y forma) de los informes } \\
\hline \multicolumn{6}{|l|}{$\begin{array}{l}\text { Temática observada de interés y relevancia en la actividad diaria del } \\
\text { desempeño docente }\end{array}$} \\
\hline \multicolumn{6}{|l|}{ Normas A.P.A. (tanto en el texto como en las referencias) } \\
\hline \multicolumn{6}{|l|}{$\begin{array}{l}\text { Trabajo en grupo, asistencia a clases obligatorias, reparto de tareas y } \\
\text { organización }\end{array}$} \\
\hline \multicolumn{6}{|l|}{ Título } \\
\hline \multicolumn{6}{|l|}{ Es claro y conciso } \\
\hline \multicolumn{6}{|l|}{ Describe los contenidos de la investigación } \\
\hline \multicolumn{6}{|l|}{ Revisión de la literatura y planteamiento del problema } \\
\hline \multicolumn{6}{|l|}{$\begin{array}{l}\text { Integra y analiza las aportaciones de los diferentes autores sobre la } \\
\text { temática }\end{array}$} \\
\hline \multicolumn{6}{|l|}{$\begin{array}{l}\text { Los argumentos son fundamentados siguiendo una perspectiva } \\
\text { analítica y crítica }\end{array}$} \\
\hline \multicolumn{6}{|l|}{$\begin{array}{l}\text { Se especifica claramente la pregunta a la que se quiere dar respuesta } \\
\text { con la observación. } \\
\text { Metodología }\end{array}$} \\
\hline \multicolumn{6}{|l|}{ Aparece una descripción de los sujetos de investigación } \\
\hline \multicolumn{6}{|l|}{ Se especifican las características del contexto a observar } \\
\hline $\begin{array}{l}\text { El procedimiento de análisis de datos es adecuado en función de las } \\
\text { características de la investigación }\end{array}$ & & & & & \\
\hline
\end{tabular}




\begin{tabular}{|c|c|c|c|c|c|}
\hline \multirow{2}{*}{ DIMENSIONES } & \multicolumn{5}{|c|}{ VALORACIÓN } \\
\hline & 1 & 2 & 3 & 4 & 5 \\
\hline \multicolumn{6}{|l|}{ Resultados } \\
\hline \multicolumn{6}{|l|}{$\begin{array}{l}\text { Los resultados presentados son congruentes con el interrogante de } \\
\text { investigación }\end{array}$} \\
\hline \multicolumn{6}{|l|}{$\begin{array}{l}\text { Los resultados están completos, cuantitativos (presentándose por un } \\
\text { lado los gráficas y tablas con sus respectivos comentarios), y por otro } \\
\text { el proceso de categorización de los datos cualitativos del apartado de } \\
\text { abierto de observaciones. } \\
\text { Conclusiones }\end{array}$} \\
\hline $\begin{array}{l}\text { Aparecen detallados los hallazgos más relevantes de la investigación y } \\
\text { se incluye alguna cita al respecto. }\end{array}$ & & & & & \\
\hline Valoración Global & & & & & \\
\hline
\end{tabular}

Cuadro 1. Ponderación del producto de la tarea 1

\begin{tabular}{llcc}
\hline Producto tarea 1 (IOS) & \multicolumn{2}{c}{$\begin{array}{c}\text { Ponderación } \\
\text { Tarea }\end{array}$} & Asignatura \\
\hline Informe de observación sistemática aplicada en un & $60 \%$ & \\
centro/contexto de Educación Infantil & & $30 \%$ \\
Evaluación profesorado & $80 \%$ & $30 \%$ \\
Autoevaluación* & $20 \%$ & \\
Total & & $100 \%$ & \\
\hline
\end{tabular}

* La calificación referente a la autoevaluación se tendrá en cuenta siempre que no exista una diferencia de 15 o más puntos (1,5 puntos sobre 10) respecto a la evaluación realizada por el profesorado.

\section{Breve Cv de los autores}

\section{Jaione Cubero Ibáñez}

Doctora en Ciencias Sociales y Jurídicas por la Universidad de Cádiz. Profesora Ayudante Doctor en el Área de Métodos de Investigación y Diagnóstico en Educación. Miembro de la Cátedra UNESCO - Evaluación, innovación y excelencia en educación y del Grupo de Investigación Interuniversitario EVALfor SEJ 509 - Evaluación en contextos formativos. Sus principales líneas de investigación son la evaluación educativa y el desarrollo competencial. Ha participado en distintos proyectos de investigación a nivel autonómico, nacional, europeo y de cooperación internacional. Coautora de varias publicaciones y comunicaciones a congresos nacionales e internacionales sobre evaluación en el contexto universitario. Formadora en evaluación y recursos tecnológicos para la evaluación. ORCID ID: https://orcid.org/0000-0002-3280-8229. Email: jaione.cubero@uca.es

\section{Nicolás Ponce González}

Doctor en Ciencias de la Educación por la Universidad de Granada. Profesor Sustituto Interino en el Área de Métodos de Investigación y Diagnóstico en Educación en la Universidad de Cádiz. Ha participado en proyectos de investigación a nivel autonómico e Internacional. Miembro del grupo de trabajo del Proyecto FLOASS I+D+i. Sus principales líneas de investigación son formación para el desarrollo de competencias interculturales, evaluación de programas de intervención en inteligencia emocional y 
evaluación educativa. Coautor de varias publicaciones y comunicaciones a congresos nacionales e internacionales sobre Investigación Educativa, Interculturalidad e Inteligencia Emocional. ORCID ID: https://orcid.org/0000-0003-1336-6303. Email: nicolas.ponce@uca.es 